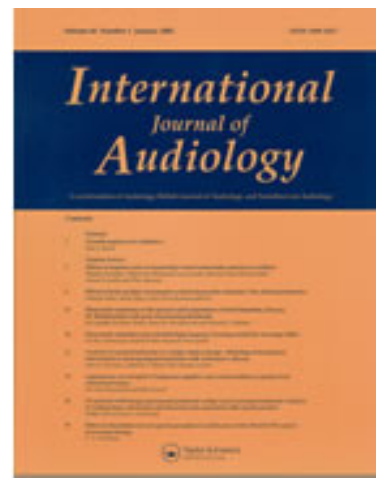

\title{
The TinMan study: feasibility trial of a psychologically informed, audiologist-delivered, manualised intervention for tinnitus
}

\begin{tabular}{|c|c|}
\hline Journal: & International Journal of Audiology \\
\hline Manuscript ID & TIJA-2018-12-0381.R2 \\
\hline Manuscript Type: & Original Paper \\
\hline $\begin{array}{r}\text { Date Submitted by the } \\
\text { Author: }\end{array}$ & $\mathrm{n} / \mathrm{a}$ \\
\hline Complete List of Authors: & $\begin{array}{l}\text { Taylor, John; University of Nottingham, NIHR Nottingham Biomedical } \\
\text { Research Centre, Hearing Sciences, Division of Clinical Neuroscience, } \\
\text { School of Medicine } \\
\text { Thompson, Dean; University of Birmingham, Institute of Applied Health } \\
\text { Research } \\
\text { Hall, Deborah; University of Nottingham, National Institute for Health } \\
\text { Research Nottingham Biomedical Research Centre } \\
\text { Walker, Dawn-Marie; University of Southampton, Health Sciences } \\
\text { McMurran, Mary; University of Nottingham, Division of Psychiatry and } \\
\text { Applied Psychology } \\
\text { Casey, Amanda; Aston University, School of Life and Health Sciences } \\
\text { Featherstone, Debbie; Clitheroe Therapies Clinic, Clitheroe } \\
\text { MacDonald, Carol; University of Stirling, Psychology } \\
\text { Stockdale, David; British Tinnitus Association, British Tinnitus } \\
\text { Association } \\
\text { Hoare, Derek; University of Nottingham, NIHR Nottingham Biomedical } \\
\text { Research Centre, Hearing Sciences, Division of Clinical Neuroscience, } \\
\text { School of Medicine }\end{array}$ \\
\hline Keywords: & Tinnitus, Psycho-social/Emotional, Treatment manual, RCT \\
\hline
\end{tabular}

\section{SCHOLARONE ${ }^{\text {m }}$ Manuscripts}




\title{
The TinMan study: feasibility trial of a psychologically informed, audiologist-delivered, manualised intervention for tinnitus
}

\author{
John A Taylor*1,2, Dean M Thompson ${ }^{3}$, Deborah A Hall ${ }^{1,2, \#, ~ D a w n-M a r i e ~ W a l k e r ~}{ }^{4}$, Mary \\ McMurran $^{5}$, Amanda Casey ${ }^{6}$, Debbie Featherstone ${ }^{7}$, Carol MacDonald ${ }^{8}$, David Stockdale ${ }^{9}$, \\ Derek J Hoare ${ }^{1,2}$
}

*authors made an equal contribution

${ }^{1}$ National Institute for Health Research (NIHR) Nottingham Biomedical Research Centre, 113 The Ropewalk, Nottingham, NG1 5DU, UK

${ }^{2}$ Hearing sciences, Division of Clinical Neuroscience, School of Medicine, University of Nottingham, Nottingham, NG7 2UH, UK

${ }^{3}$ Institute of Applied Health Research, University of Birmingham, Edgbaston, Birmingham, B15 2TT, UK

${ }^{4}$ Health Sciences, University of Southampton, Southampton, SO17 1BJ, UK

${ }^{5}$ Division of Psychiatry \& Applied Psychology, University of Nottingham, NG7 2UH, UK

${ }^{6}$ School of Life \& Health Sciences, Aston University, Birmingham, B4 7ET, UK

${ }^{7}$ Clitheroe Therapies Clinic, 3 Castlegate, Clitheroe, Lancashire, BB7 1AZ, UK

${ }^{8}$ Department of Psychology, University of Stirling, Stirling, FK9 4LA, UK

${ }^{9}$ British Tinnitus Association, Ground Floor, Unit 5, Acorn Business Park, Woodseats Close, Sheffield, S8 0TB, UK

\# current address: University of Nottingham Malaysia, Jalan Broga, 43500 Semeniyh, Selangor Darul Ehsan, Malaysia

Correspondence: derek.hoare@nottingham.ac.uk 


\begin{abstract}
Objective: To develop a manualised psychological treatment for tinnitus that could enhance audiologist usual care, and to test feasibility of evaluating it in a randomised controlled trial. Design: Feasibility trial, random allocation of patients to manualised treatment or treatment as usual, and mixed-methods evaluation.
\end{abstract}

Study sample: Senior audiologists, and adults with chronic tinnitus.

Results: Recruitment reached 63\% after 6 months (feasibility pre-defined as 65\%). Only nine patients $(47 \%)$ were retained for the duration of the trial. Patients reported that the treatment was acceptable and helped reassure them about their tinnitus. Audiologists reported mixed feelings about the kinds of techniques that are presented to them as 'psychologically informed'. Audiologists also reported lacking confidence because the training they had was brief, and stated that more formal supervision would have been helpful to check adherence to the treatment manual.

Conclusions: The study indicate potential barriers to audiologist use of the manual, and that a clinical trial of the intervention is not yet feasible. However, positive indications from outcome measures suggest that further development work would be worthwhile. Refinements to the manual are indicated, and training and supervision arrangements to better support audiologists to use the intervention in the clinic are required.

Trial Registration:ISRCTN13059163. 
Keywords: Tinnitus, Audiology, Psychological intervention, Randomised controlled trial (RCT), Manual 


\section{Background}

2 Tinnitus involves the perception of sound in the absence of any acoustic stimuli (Davis \& El

3 Refaie, 2000), and is often associated with insomnia, hearing problems, depression, and

4 anxiety (Tyler \& Baker, 1983; Pinto et al., 2014). Cognitive behaviour therapy (CBT) has

5 been trialled extensively, but evidence is largely limited to clinical trials where a psychologist

6 delivered it (Fuller et al., 2020; Hesser et al., 2011; Hoare et al., 2011). Some studies have

7 additionally included multiple disciplines such as clinical physicists in audiology, who

8 deliver elements of care that are informed by psychology (e.g. Cima et al., 2012). In the UK

9 most tinnitus care is delivered by audiologists. However, there is no standard protocol for

10 tinnitus management and various approaches to assessment and treatment are used (Hoare et

11 al., 2015). Most audiology departments offer management of hearing loss, use of sound

12 generators, and patient education. Only some departments have access to psychologists

13 (Gander et al., 2011) and there is no evidence for the effectiveness of psychological

14 interventions when delivered by audiologists (Hoare et al., 2011). If audiologists were to use

15 a psychologically informed treatment approach, this could lead to improved outcomes and a 16 more cost-effective service (Stockdale et al., 2017; Wan Suhailah et al., 2015).

18 The Department of Health (now the Department of Health and Social Care) (2009)

19 recommends that audiologists provide psychological therapies where psychologists are not 20 available. Furthermore, patients and clinicians agree that whether CBT delivered by 21 audiologists is an effective treatment for tinnitus is a priority research question (Hall et al., 22 2013). To that end, and in accordance with Medical Research Council (MRC)

23 recommendations for developing and evaluating complex interventions (Craig et al., 2008),

24 we took a phased approach to developing an audiologist delivered intervention that was

25 'psychologically informed'. To begin with, components of psychological therapies for 
tinnitus were catalogued in a scoping review (Thompson et al., 2017). The primary aim of

27 that scoping review was to catalogue components of psychological therapies which have been

used or tested by psychologists in a format that would inform the development of a

standardised audiologist-delivered psychological intervention. Twenty-five 'themes' of

components were identified including tinnitus education, psychoeducation, evaluation,

treatment rationale, treatment planning, problem solving, behavioural intervention, thought

identification, thought challenging, worry time, emotions, social comparison, interpersonal

skills, self-concept, lifestyle advice, acceptance and defusion, mindfulness, attention,

relaxation, sleep, sound enrichment, comorbidity, treatment reflection, relapse prevention,

and common therapeutic skills.

In the second development stage, consensus on which therapy components audiologists

should deliver as part of their usual care was derived in a three-round Delphi survey of

patients who have experienced tinnitus counselling or psychological therapy, and specialist

audiologists, hearing therapists, and psychologists, who had significant experience of using

counselling or psychotherapeutic techniques for tinnitus (Thompson et al., 2018). The Delphi

survey included all those components identified in the scoping review (Thompson et al.,

2017) and any additional components identified by our experts during the first round of the

survey. Components that typically reached consensus for inclusion were common therapeutic

skills such as Socratic questioning and active listening, which are common to different

therapeutic modalities, rather than specific techniques such as graded exposure therapy or

cognitive restructuring, which are unique to a cognitive behavioural orientation. There was model in patient education. 
51 Here we report the next stage of this work. The aims were to (1) manualise a low-intensity audiologist-delivered psychologically informed intervention for tinnitus, and (2) evaluate

53

54

55 56

57 feasibility of delivering and evaluating the manualised intervention. The intervention was delivered by NHS audiologists with prior experience in tinnitus management. The study primarily considered feasibility of a clinical trial of the manualised intervention in terms of the likely patient population, willingness of patients to receive/engage with the manualised treatment, willingness of audiologists to recruit and randomise tinnitus patients to a trial, and willingness of patients to be randomised. Secondary objectives considered qualitative judgements on the acceptability of the intervention from audiologist and patient perspectives, and the likely completeness of selected outcome measures in a randomised controlled trial (RCT). It was our aim to embed good practice in patient and public involvement at all stages.

\section{Methods}

The protocol for this work has previously been published (Taylor et al., 2017), and is summarised here.

\section{Development of the manual}

Manual development commenced with a one-day reference group meeting involving steering group and study team members (patients, audiologists, hearing therapists, psychologists, and researchers) to decide on which components to include, and how to incorporate them into a manualised care protocol. It was agreed at this meeting that a working framework for the manual would comprise the following sections: Rationale, Assessment, Education, Treatment Planning/Goal Setting, Management/Self-Management, and Relapse Prevention. All 76 components for which consensus for inclusion was reached in the Delphi survey (Thompson et al., 2018) were first considered for allocation under these headings. This was followed by a 
76 discussion on whether any of the 84 components for which consensus was not reached should

77 be included (no component reached consensus to absolutely exclude) and if so, under which headings they would be written into the manual. Decisions were based on (1) level of agreement from the Delphi survey, (2) whether components had a good evidence-base, 80 theoretical cohesiveness, and, (3) what could realistically be included in a brief low-intensity intervention delivered by audiologists given resource limitations and time-limited training. Individual members of the team drafted specific sections of the manual in accordance with their areas of expertise and according to consensus opinion on what features are typical of a 'good' treatment manual (McCulloch \& McMurran, 2007). The manual was then reviewed and amended to reflect a 'whole' in terms of style, coherence, and theory. contained in the manual) detailing background and overview of the manual, assessment through a standardised interview, the rationale for psychologically informed treatment, collaborative goal setting and treatment planning, tinnitus education, management/selfmanagement strategies, relapse prevention, a bibliography containing references related to the intervention and further reading for the audiologist or patient, and appendices containing materials used for training purposes. An accompanying 'toolkit' (listed in Supplemental Information 2) of questionnaires, worksheets, and information leaflets was collated to support use of the manual.

\section{$97 \quad$ Feasibility trial}

98 Ethical approval for the feasibility trial was granted by North West - Preston Research Ethics

99 Committee (reference: 16/NM/0047). The trial involved random allocation of tinnitus

100 patients who received psychologically informed treatment from an audiologist trained in the 
101

102

103

104

105

106

107

108

109

110 Trial sites and training

111 Patients were recruited at three sites, audiology/Ear, Nose and Throat (ENT) services situated

use of the manual, or treatment as usual (TAU) from an audiologist who was not trained in or aware of the content of the treatment manual (Figure 1). Patients were randomised using the randomisation function in Microsoft Excel such that patients allocated to the greatest five random numbers per site were allocated to TAU. A member of the research department not involved in the trial performed randomisation. There was no blinding (to allocation) of the researchers who collected or analysed the qualitative or quantitative data.

\section{***INSERT FIGURE 1 ABOUT HERE***} in (1) Nottingham University Hospitals NHS Trust, (2) Sherwood Forest Hospitals NHS

113 Foundation Trust, and (3) Derby Hospitals NHS Foundation Trust. The audiologists

114 delivering the psychological tinnitus intervention and TAU at each site were senior

115 audiologists with several years of experience of managing tinnitus. A 2-day workshop was 116 developed by AC and DF to train audiologists to deliver the manual. Training was designed

117 to be interactive by using a constructive alignment approach (Biggs, 2003). AC developed

118 lesson plans using the Race lecturer toolkit (Race, 2014) including learning outcomes for 119 each session; resources needed to deliver the session; and the structure, content and method 120 of delivery. One senior audiologist from each site received training in the use of the manual 121 during the workshop. Learning was assessed using the Teach-back technique (Meyer et al., 122 2010).

\section{Participants}


125 The recruitment method targeted people with tinnitus attending each participating site for an 126 audiology assessment appointment as part of the NHS standard care. Following consultation

127 with six NHS audiology sites, it was estimated that on average recruitment of two eligible 128 patients per month per site was readily achievable. We therefore conservatively estimated 129 feasibility of a trial to reflect this and requested each site to recruit and randomise up to ten 130 patients within a 6 month period. For inclusion, patients were required to (1) be adults aged 13118 and over, (2) have intellectual/cognitive capacity to provide informed consent, (3) have 132 sufficient mobility to attend clinics, (4) score $\geq 25$ on the Tinnitus Functional Index (TFI; 133 Meikle et al., 2012), and (5) be willing to complete questionnaires and a semi-structured 134 interview about their experiences of taking part in the trial. Patients were excluded if they (1) 135 had tinnitus with a medically treatable origin, (2) were unable to communicate in English, (3) 136 had comorbid conditions (e.g. dementia) which meant they were unable to give informed 137 consent, or (4) had participated in other tinnitus management research between consenting 138 and the start of the intervention. No exclusions were made because of previous experience 139 with psychological services or medications taken. All patients were enrolled at or before their 140 first visit to audiology with a primary complaint of tinnitus.

142 A member of the research team (JAT) was responsible for recruitment at Site 1. At Site 2 and 143 Site 3, the local PI was delegated responsibility for recruitment, with infrastructure support 144 provided by the National Institute for Health Research (NIHR) Clinical Research Network. 145 JAT regularly monitored recruitment at Sites 2 and 3 and maintained regular email contact 146 with the local PIs to offer support where recruitment rate was lower than required. However, 147 no additional support was requested during the trial. 
150 Psychologically informed treatment was delivered according to the treatment manual. This 151 involved an average of 2.75 sessions (range $=1-3$ ) delivered one-to-one by an audiologist 152 who attended the training workshop.

153

154 Control

155 Patients allocated to the control condition received TAU from an audiologist not trained in 156 psychological therapy and who had not received training in the manualised intervention.

157 TAU typically involved management of hearing loss, education and advice, lasting an 158 average of 1.5 sessions (range $=1-2$ ).

159

160 Outcomes

161 Feasibility of a powered RCT was predefined as (1) a recruitment efficiency of at least 10\%

162 of those screened, (2) recruitment of at least $65 \%$ of the overall target sample size, (3)

163 retention of $80 \%$ of patients who were randomised, (4) completion of $80 \%$ for the primary

164 outcome questionnaires, and (5) patient and clinician compliance to the manualised tinnitus

165 counselling.

166

167 Six questionnaire were completed at each time point. The TFI is a measure of tinnitus

168 symptom severity over the last 2 weeks. It has 25 items (e.g. "Did you feel IN CONTROL in 169 regard to your tinnitus?"), each with a 10-point scale. Scores are summed and divided by 2.5 170 to give a total possible score between 0 and 100. The eight factor TFI has very high internal 171 consistency $(\alpha=0.97)$ (Meikle et al., 2012). It has excellent reliability, stability over time, 172 and sensitivity to individual differences in tinnitus severity, although the auditory subscale 173 (three items) may not contribute to the overall score (Fackrell et al., 2018). 
175 The Tinnitus Cognitions Questionnaire (TCQ; Wilson \& Henry, 1998) consists of a series of 176 statements preceded by the words "I think" or "I tell myself," (e.g., I think "if only the noise 177 would go away"). The first 13 items refer to negative thoughts and the second 13 items refer 178 to positive thoughts. Responses are marked on a five-point Likert scale from "never" = 0, to 179 "very frequently" $=4$. Positive items are reverse scored to give a total score from $0-104$. The 180 TCQ has a reliable two-factor structure and high internal consistency $(\alpha=0.90)$ (Handscomb 181 et al., 2017).

182

183 The Clinical Outcomes in Routine Evaluation - Outcome Measure (CORE-OM; Barkham et 184 al., 2006) is a 34-item (four factor) measure of global distress. Respondents indicate how 185 much each item (e.g. "I have felt optimistic about my future") has applied to them over the 186 past week, using a 5-point Likert scale from "not at all" to "most or all of the time". The CORE-OM includes both positive and negative items (positive items being reverse scored) to 188 give a total score from $0-136$. Initial validation of this four factor questionnaire found it to 189 have high internal consistency ( $\alpha=0.94$ ) (Evans et al., 2002), although validation in a 190 tinnitus population found that a three factor structure provided a better fit to the data 191 (Handscomb et al., 2016).

193 The Health Utilities Index 15Q (HUI; Furlong et al., 2001) is a 15 item measure of health194 related quality of life. Items are multiple choice with 5-6 rank-ordered response options.

195 Decision tables of response combinations are used to determine the health-state level for each 196 domain within the questionnaire, and then using the tables and a scoring algorithm, utility

197 scores for all attributes of health and an overall health-related quality of life score is 198 determined. Overall scores range from $-0.36-1.00$, where $0=$ "death" and $1=$ "perfect 199 health”. Estimates of the reliability of the HUI vary considerably (Busija et al., 2011). 
201 The Working Alliance Inventory (WAI; Horvath \& Greenberg, 1989) provides a measure of 202 the quality of alliance within counselling. It contains 36 items (e.g. "I was worried about the 203 outcome of the sessions") scored on a Likert scale from 1 (Never) to 7 (Always). The WAI 204 contains both positive and negative items, with negative items being reversed scored, giving a 205 possible range of scores from 36 to 252 . Reliability of the three WAI scale scores appear to 206 be high ( $\alpha=0.92,0.92$, and 0.89 respectively) although two subscales ("Tasks" and "Goals") 207 were very highly correlated $(\mathrm{r}=.92)$. 208

209 The Client Service Receipt Inventory (CSRI; Beecham \& Knapp, 2001) is an adaptable form 210 used to collect information on the whole range of services and supports study participants 211 may use. It contains items such as "Please list any use of inpatients hospital services over the 212 last three months".

213

214 After randomisation, and in advance of their first audiology appointment, patients completed 215 the baseline study questionnaires. Questionnaires were completed again immediately at the 216 end of treatment and at follow-up or 6 months after the commencement of treatment 217 (excluding the CSRI which was only completed at follow-up). In practice, some 6-month 218 follow-up questionnaires were completed before the end of treatment. Patients received one 219 reminder (telephone or email) to complete the follow-up questionnaires if they had not been 220 returned within 2 weeks of their due date.

221

222 Treatment fidelity

223 Treatment fidelity required that treatment components were all consistency administered 224 according to the manual. Audiologists recorded administration of treatment components in a 
225

226

227

228 229

case report form held for each patient, and took part in semi-structured interviews after the last patient had completed their treatment. Interviews were used to determine which components of the manualised care they thought worked well, why, and which components were not useful. Patients involved in the intervention group also took part in semi-structured interviews to discuss their experiences of the intervention. JAT or DMT conducted interviews approximately 4 weeks after the last patient appointment. Clinician interviews were conducted after discharge of their final patient. Interviews were audio-recorded, transcribed verbatim and analysed using a thematic analysis approach (Braun \& Clarke, 2006). DMT, DMW, and two patient representative members of the lead centre's Patient and Public Involvement (PPI) panel conducted analysis of the qualitative data.

\section{Deviations from protocol}

We had originally planned that public research partners trained in interview techniques would conduct patient interviews. However, this ultimately proved unworkable due to the Sponsor's stipulation that they would need formal registration as volunteers with the NHS Trust for this activity. The process for this, involving application and interviews, was deemed too time consuming to be accommodated within the study timeline. We had also originally planned to discuss emerging themes in two focus groups with audiologists and patients, to ensure that the themes and any identified barriers and facilitators to treatment implementation and maintenance of self-management were accurately representative of patient and audiologist experience. However, given the unanticipated length of the treatment process at sites, there was insufficient time for this to be included within the funding period.

\section{Results}

\section{Demographic and clinical characteristics}


250 Nineteen patients were recruited to the trial and randomised to manualised care $(n=11)$ or 251 TAU $(n=8)$. Demographic characteristics are reported in Table 1. Mean baseline TFI score 252 was $67.08(\mathrm{SD}=23.644)$. Seven patients scored 25-50 on the TFI, scores interpreted as 253 tinnitus being a significant problem with a possible need for treatment (Henry et al., 2014). 254 Eleven patients scored above 50, interpreted as tinnitus severe enough to qualify for treatment 255 that is more intensive. Demographic data were missing for one patient. 256 257 ***INSERT TABLE 1 ABOUT HERE*** 258 259 Feasibility outcomes

260 Participant flow is presented in Figure 1, and feasibility outcomes are presented in Table 2. 261 Patients were screened from September 2016 to April 2017 (maximum six months from first 262 screen at each site). At Site 1, all 10 participants were recruited within a 3 month period; at 263 Site 2 three patients were recruited within a 3 month period, two of whom were recruited on 264 the same day); at Site 3 six patients were recruited within a 7 week period, five of whom 265 were recruited within the same week). 266

267 Six-month follow-up occurred from April to September 2017. Recruitment efficiency of 268 those screened surpassed the feasibility target at Site 1 where 10 eligible patients were 269 enrolled from just 16 patients screened (63\%), while the target was one enrolment for every 27010 patients screened (10\%). Recruitment efficiency was also met at Site 2, where 24 patients 271 were screened and three patients were recruited (13\%). This difference in recruitment 272 efficiency is in part a consequence of different patient pathways; at Site 1 all patients were 273 referred by their GP to ENT, and from ENT onto audiology. At Site 2 some patients were 274 referred directly to audiology by their GP. Indeed 10 of the 24 patients screened at Site 2 
275 were assessed by the audiologist as requiring referral to ENT or psychiatry and therefore not 276 eligible for the trial. Also at Site 2, one recruited patient failed to attend any further 277 appointments, and three patients screened as eligible declined to participate due to the time 278 commitments involved and geographical distance between home and the audiology service. 279 Site 3 did not return their screening records so we were unable to assess feasibility. 280 281 ***INSERT TABLE 2 ABOUT HERE*** 282 283 Nineteen patients were recruited and consented to take part in the study; representing a total 284 recruitment of $63 \%$ (feasibility target was $65 \%$ ). Site 1 surpassed the target for feasibility 285 with $100 \%$ recruitment. Site 2 recruited three patients $(30 \%)$ and Site 3 recruited six $(60 \%)$. 286 287 288 289 290 291 further $(n=1)$, or commenced other treatment $(n=3)$.

\section{Effectiveness outcomes}

Completeness of questionnaire data is given in Table 2, and descriptive statistics are 296 presented in Table 3. TFI, TCQ, TCQ negative thoughts subscale, and CORE-OM scores 297 decreased between baseline and the end of treatment. TFI scores continued to decrease 298 between the end of treatment and 6-month-follow-up, whereas TCQ and CORE-OM scores were maintained between the end of treatment and 6-month follow-up. TFI emotional distress 300 subscale scores increased between baseline and the end of treatment, but decreased below 301 baseline by 6-month-follow up. Patients and audiologists' scores on the WAI task, bond, and 
302 goal subscales all increased between baseline and end of treatment. No adverse events or 303 adverse reactions were recorded during the trial.

304

305

306

307

308

309

310

311

312

313

314

315

316

317

318

319

320

321

322

323

324

\section{***INSERT TABLE 3 ABOUT HERE***}

\section{Treatment fidelity and experience}

Six themes emerged from thematic analysis of interview transcripts: (1) acceptability of and fidelity to treatment components, (2) factors affecting fidelity, (3) refining treatment components, (4) factors affecting feasibility of wider implementation of treatment, (5) training and supervision, and (6) measurement of tinnitus outcomes.

(1) Acceptability of and fidelity to treatment components.

Patients and audiologists use, acceptance of, and fidelity to the treatment components varied. All audiologists reported that they actively promoted physical activity, and patients reported that they engaged in physical activity during the trial, despite it temporarily increasing awareness of tinnitus. Other components were not used consistently, e.g. one audiologist and one patient (from different sites) stated that they did not formally set any goals for treatment, thereby deviating from the treatment protocol:

“We didn't set any formal goals, it was just a case of, I would go away and see... whether it's got any worse or whether it got any better and in actual fact it's stayed about the same." (Patient 2).

Another audiologist explained that goal setting can be challenging without first engaging in tinnitus education to explain to the patient that they cannot cure their tinnitus: 
325

326

327

328

330

331

332

333

336

337

339

340

341

342

343

344

345

346

347

348

349

“... I find the preliminary bit of goal setting a bit difficult because you've got to start by saying, 'we can't cure your tinnitus, so with that in your mind, what would you like to do? "’ (Audiologist 3).

All patients reported being reassured by receiving information about the causes, maintenance, and change in tinnitus over time, and for some patients, education was considered sufficient intervention. For many patients the cognitive behavioural model of tinnitus reflected their personal experiences of tinnitus. Patients and audiologists also commented that the model was understandable:

I think [the cognitive behavioural model is] quite a nice one because it's quite logical, the language that it uses is fairly easy, is nothing too sort of technical or too medical and you can [work] through [the manual] in a logical way." (Audiologist 2)

Relaxation was acceptable to audiologists although it was used sparingly and briefly in and outside of appointments. Patients mostly reported using relaxation to improve sleep. Patients and audiologists also highly valued the inclusion of education about sleep cycles in treatment. Patients in the trial did not use sleep diaries, sleep monitoring devices were favoured, although the principle of monitoring sleep to identify poor sleeping habits was maintained: "No, I've been using [a fitness watch and not the sleep diary] because that records in quite reasonable detail the amount of sleep you've had." (Patient 1)

Cognitive techniques received mixed reviews. Some patients expressed an understanding of avoidance as a mechanism of tinnitus-related distress and recognised when they were engaging in avoidance behaviours:

"I avoided noisy environments. So, like if friends invited me round for a party, I always used to make excuses not to go..." (Patient 3)

Audiologists also demonstrated an understanding of safety behaviours such as avoidance. However, at least one felt unable to recognise avoidance consistently in various contexts: 
350

351

"Safety [avoidance] behaviours...seems very obvious when you read the words.... But then actually when the patient starts talking about the things that they do and their feelings and their attitudes and how they deal with things you don't necessarily think, 'that's a safety behaviour'." (Audiologist 1)

At least one audiologist found thought challenging prompts included in the treatment manual useful, but fidelity to the thought-challenging component varied, often limited to identifying negative thoughts without challenging them:

"I didn't use the thought record sheets. I used the unhelpful thinking styles [material], and patients do find this one quite useful and quite often they'll point out, 'yeah, that's me', and so that helps us to address some of these thoughts." (Audiologist 3)

When thought challenging was used, audiologists either considered the technique ineffective, or believed the benefit would only be short term:

“Most of the time [patients] don't [think of a more helpful realistic thought]. And I don't know whether that's because I'm not enabled enough or whether they just can't do it." (Audiologist 1)

The attention, monitoring and acceptance component of the manual was used in treatment but not consistently or as instructed in the manual. One audiologist introduced the concept of mindfulness, and two used metaphors when they thought it would be acceptable to patients. However, one patient reported their audiologist used metaphors as an imagination exercise rather than as an (intended) defusion technique:
“We didn't go through too many metaphors, obviously the metaphors of aligning the sounds of your tinnitus to natural sounds. We didn't talk about particular river banks." (Patient 1) 
373 Finally, relapse prevention was not addressed according to the manual. Patients remembered

374 being advised to return if their symptoms worsened or if self-management using the

375 techniques learned during the treatment were not effective:

376

"I don't think [the audiologist] did [share the relapse prevention handout] actually, no because I don't think we got on to that point actually." (Patient 1)

378

379 (2) Factors affecting fidelity

380 Audiologists reported a lack of confidence in implementing treatment components including 381 relaxation, acceptance-based components, and goal setting. One suggested that rigid use of 382 the manual would impair the development of the therapeutic relationship, particularly for less 383 experienced audiologists, and in contrast, another felt that the manual would help less experienced audiologists by providing a structure to follow. For one audiologist, confidence reportedly augmented with increasing experience during the trial:

"You're always a little bit nervous to start with. However, I found that after the first few appointments it's just flown. You develop your own pattern in appointments and I guess I developed a pattern very, very quickly and that's because it was very intense training..." (Audiologist 3)

Patients sometimes resisted the use of treatment components such as thought challenging or mindfulness. Audiologists stated their patients would report not having enough time to engage with the treatment outside of appointments, or just believed that the treatment would 393 be unsuccessful. Audiologists also considered some treatment components were within the scope of practice of psychologists or hearing therapists, but not theirs:

"To me [thought challenging] is a logical skill and I don't know that that's my leaning at all. To say to somebody, 'well that thought you are having there is very unhelpful'....if you're a psychologist you could do that. But I can't." (Audiologist 1) 
399 (3) Refining treatment components

400 Patients and audiologists recommended changes to the manual. These included expanding the 401 physical exercise component to include lifestyle advice on diet and alcohol. "That's something that we do anyway so within the tinnitus therapy process we 403 always talk about lifestyle choices. So, physical exercise comes into that but we also 404 talk about things like diet, we might also talk about you know where patients are 405 maybe drinking excessively." (Audiologist 3) "If they've taken up hobbies that have enabled them to be more distracted, sort of reinforcing those things and said, 'these are the things that you know are going to enable you in the long term to sort of ignore your tinnitus and live with it happily'."

410 One audiologist found that some patients' lack of motivation was a barrier to their 411 engagement in the treatment and that a technique to improve motivation would be useful. “It's like how can we break this down... what happens when you can't motivate a patient... how do you tackle somebody who is clearly giving you excuses...? Cause there's a harsh way which is, 'look, you're just giving lots of excuses.' But that's not the way to do it. That doesn't help anybody. But then is it up to us to go into maybe the deeper reasons why they're doing that, is it a confidence issue [for example], and it's that thing that's stopping that patient from getting better." (Audiologist 3)

418 (4) Factors affecting feasibility of wider implementation of treatment

419 Audiologists unanimously agreed the treatment did not lengthen the amount of time spent 420 with patients throughout their care. However, participation in the intervention arm of the trial 421 affected the usual appointment structure. For example, the audiologists delayed hearing aid 422 fitting to allow time to gather information about underlying mental health problems first. 
423 Audiologists also thought that reducing the time between appointments could increase 424 treatment benefit:

425

“[reducing] follow-up [from] about 12 .. to...6 weeks after the initial appointment... works because patients either take on board everything you say or they struggle with it and if you've kind of let them go for three months you're almost back to square one sometimes." (Audiologist 1)

However, concern was expressed that such changes to appointment structure might not be feasible in audiology services where resources are limited. Continuity of care, with patients seeing the same audiologist throughout their care was also considered beneficial: “.. some patients even though they are hearing aid patients, probably would benefit from being fitted by the tinnitus audiologist rather than an [other] audiologist because some of the reasoning behind why we're fitting the hearing aid has to be related to the tinnitus. And perhaps that was lost ..." (Audiologist 2)

(5) Training and supervision

Audiologists were introduced to the manual across 2 days of training. Although audiologists valued the small group setting, the content of the 2 days was considered overwhelming. Audiologists involved in the trial suggested that it might not be feasible to implement this format outside of the trial for routine clinical practice:

"It was excellent, one-to-one with a group of experts, what else could you ask for? ...Feasibility-wise, would that work in practice? Probably not." (Audiologist 3)

Some audiologists recommended role-play to improve the training to provide practice and feedback on techniques in a range of scenarios in order to help audiologists to understand how components of the manual would work in practice. All audiologists felt that formal 
447

448

449

450

451

452

453

454

455

456

457

458

459

460

461

462

463

464

465

466

467

468

469

470

471

supervision arrangement would be helpful to check adherence to the treatment manual.

However, audiologists also noted the lack of supervision within audiology:

"In adult audiology, we work in a silo... we don't have anyone else except our colleagues to turn to, we don't have any formal supervision like you would if you were a therapist." (Audiologist 3)

The potential for the treatment manual to be used as a training tool for inexperienced audiologists was raised repeatedly during interviews:

"I found [the manual] quite useful in terms of training somebody new to sort of say, 'do you want to delve a bit deeper, is there something in these questions that perhaps is relevant for the patient you've got sat in front of you?" (Audiologist 2)

(6) Measurement of tinnitus outcomes

Patients and audiologists recognised that all the domains measured by the TFI and TCQ could be relevant to different patients, and no additional domains were identified as relevant that were not measured in the trial. The TFI was favoured as an assessment tool by patients and audiologists because of its broad coverage across tinnitus problem domains to enable identification of relevant treatment options. However, one audiologist highlighted that some patients conflate hearing loss and tinnitus when completing the TFI, which may limit its ability to distinguish between tinnitus-related and hearing-related distress. Audiologists were less familiar with the TCQ, although one appreciated it as a means to encourage patients to recognise and talk about their negative thoughts about tinnitus:

"Sometimes patients are quite reluctant to give [negative thoughts], some patients don't like feelings, but to get them to write it down and to score it, you get a bit more of an overview of about where they are and how severe really the tinnitus is and how much it is affecting them psychologically. So that again I've not used that one [TCQ] 
472

473

474

475

476

477

478

479

480

481

482

483

484

485

486

487

488

489

490

491

492

493

494

495

496

routinely but I do think that one is one that I would probably use for those patients where I'm perhaps struggling to see how much is affecting them psychologically." (Audiologist 2)

Patients also welcomed the use of tinnitus questionnaires to monitor their improvement during treatment:

“It makes you start to realise how well you're doing and it gives you a chance to measure that...how bad it is or how you're managing really well." (Patient 2)

Patients and audiologists had different views on the difficulty of completing questionnaires. Whereas audiologists perceived questionnaires to be accessible, patients thought that support should be offered to complete any questionnaires:

"When you give a questionnaire like this and just give it to somebody-and I was put in another room to do it - I think there's an assumption made that everyone can do that task." (Patient 2)

\section{Discussion}

This study was the first to develop and assess feasibility of trialling an audiologist-delivered low intensity psychological intervention for tinnitus. Ultimately, we aimed to provide an evidenced-based, costed, manualised approach to tinnitus care that is attractive to commissioners and represents a flexible and accessible tinnitus treatment. The feasibility trial reported here determined that a manualised psychologically informed treatment was acceptable to patients and audiologists, but that it is not yet feasible to test in a multi-site effectiveness trial. Recruitment reached only 19 patients (63\%) after 6 months, just short of the $65 \%$ target for feasibility, while average retention for the duration of the trial reached only nine patients $(47 \%)$, very short of the $80 \%$ target for feasibility. Patient recruitment is acknowledged to be one of the most difficult and least predictable elements of a clinical trial. 
497 (Allen et al., 1998; Thoma et al., 2010; Sanchez et al., 2018). Here, performance varied 498 across the three sites. The best in terms of recruitment efficiency and recruitment rate was 499 Site 1, an audiology service based in a large teaching hospital where staff are supported to be 500 research active. Having a large pool of potentially eligible patients to draw on, as well as 501 engaging with audiologists who have previous experience in participating in RCTs, are 502 known factors that can help to achieve study targets for hearing trials (Sanchez et al., 2018). 503 In addition, a member of the research team was responsible for recruitment at Site 1, and did 504 not have to contend with the same clinic demands as PIs at the other sites. That said, the 505 pattern of recruitment would suggest all could have reached feasibility. Site 3 in particular 506 recruited five patients (50\% of target) within a single week, suggesting competing demands 507 and/or a lack of engagement with the research, rather than low patient numbers, were barriers 508 to recruitment at that site. In contrast, Site 1 performed poorest in terms of patient retention 509 indicating that even experienced participating sites need a risk mitigation plan and regular 510 trial monitoring. We would concur with the recommendations suggested by Sanchez et al.

511 (2018) that a future RCT needs to ensure that it has sufficient resource to support a carefully 512 specified plan to promote recruitment and retention of tinnitus patients. Furthermore, based 513 on current findings, we would suggest that treatment fidelity could be improved and more 514 accurately monitored by (1) including an audiologist familiarisation phase of treatment 515 implementation before including patients in analyses, and (2) including direct in-session 516 observation of a random selection of treatment sessions using standardised fidelity evaluation 517 forms, based on the treatment manual.

518

519 From the Delphi survey (Thompson et al., 2018) we know that psychoeducation and common

520 therapeutic skills are considered more important than specific CBT techniques in

521 psychological tinnitus treatment. Patients and audiologists considered it essential to include 
522 psychoeducation and common therapeutic skills (aimed at informing the patient and 523 conveying empathy) in psychological tinnitus treatment. Giving patients information about 524 common cognitive distortions was considered essential, but cognitive restructuring was not.

525 This pattern suggests that patients and audiologists understood the contribution of

526 maladaptive behaviours and negative thoughts to bothersome tinnitus, but did not think that 527 the audiologist's role should or could extend to managing patients' use of specific cognitive 528 behavioural techniques. This issue was further highlighted in the current trial. Interviews 529 revealed audiologists chose not to use certain psychological treatment components from the 530 manual because (1) they lacked confidence in using them as training was brief and 531 insufficient for them to learn how to implement them safely and effectively, (2) they thought 532 patients do not want to record, monitor, and challenge specific negative thoughts and 533 behaviours, and (3) they believed certain components would be ineffective. More work is 534 therefore required to consider what audiologists can and want to deliver either within their 535 current or a revised clinic and appointment structure, and what training and supervision 536 arrangements need to be in place for these skills to be developed, used, and maintained 537 throughout a clinical trial. Beyond feasibility and evaluation of effectiveness, it will also be 538 essential to explore how the intervention can be implemented into routine clinical practice, 539 which will involve further challenges such as organisational development, workplace cultures 540 and individual barriers (De Silva, 2015).

541

542 Similar issues are being faced in other areas. For example, there is emerging evidence that 543 CBT-enhanced voice therapy for functional dysphonia leads to greater improvement in 544 general well-being and distress (Miller, Deary, \& Patterson, 2014), but as with the current 545 study there are open questions about how clinicians should be trained and supervised and 546 how cost-effective this approach may be. There are examples in other fields of how training 
547 might be achieved on a large scale. Richmond et al. (2016) evaluated feasibility of an online

548 programme to train physiotherapists in the use of cognitive behavioural approaches to the

549 management of non-specific low back pain. They found an internet-based approach to

550 training to be equivalent to face-to-face group training in terms of retention of theoretical and

551 procedural knowledge. However, clinicians who received the face-to-face training showed

552 greater self-efficacy in delivering the assessment component of the therapy, were more

553 satisfied with their training, and were more 'psychosocially' rather than 'biomedically'

554 orientated after training, and by implication were more aligned with a cognitive behavioural

555 approach. Regardless, uptake of the treatment approach after training was similar across both

556 groups (about one third). Their conclusion, that there is a need to develop strategies for

557 training and support to deliver treatments that use cognitive behavioural approaches, read

558 relevant to all allied health professionals at this time. In the context of the current study,

559 issues such as the acceptability and preference for different training formats will need to be

560 explored.

561

562 Certain revisions to the TinMan study manual and training require consideration before it can

563 be subjected to further feasibility testing. These include additional information about tinnitus

564 and psychoeducation, including information about negative thought patterns and avoidant

565 behaviours. An appreciation of patient preference and need with respect to psychological

566 treatment components was also apparent in the current study. Specific cognitive behavioral

567 techniques tend to purposefully engage patients in initially unwanted, though temporary,

568 thoughts or emotional states. It is of importance to train audiologists in how to deal with these

569 challenges and ultimately make use of the patient-states resulting from these approaches. The

570 natural resistance and transferal of distress in both patients and audiologists when using these 
571 techniques needs to be adequately covered in training that balances informed patient choice 572 and need.

573

\section{Conclusion}

575 What emerged most strongly from this study is that an audiologist-delivered low-intensity

576 psychological intervention is an acceptable approach to tinnitus management, but even very 577 experienced audiologists require more extensive training to 'upskill' in the use of 578 psychological treatment components. Audiologists involved in delivering the psychological 579 treatment reported that the amount of training was insufficient and lacked role-play and 580 feedback from a supervisor to increase understanding of how to deliver the treatment 581 effectively. This needs to be addressed before further testing. One potentially cost-effective 582 means of training audiologists may be to develop reusable learning objects, e.g. including 583 video recordings of role-play scenarios where an audiologist is using a set of psychological

584 techniques from the manual. Once training is addressed, the treatment may progress to further 585 feasibility testing. There has been recent discussion in the UK audiology community about 586 some form of compulsory continual professional development or qualification in tinnitus care 587 for practicing audiologists. The manual developed here, and the training resources that will 588 support its use, could potentially meet this perceived need. 589

\section{Acknowledgements}

591 We acknowledge the support of the National Institute for Health Research Clinical Research 592 network (CRN) in participant recruitment. Nottingham University Hospitals NHS Trust was 593 the Sponsor for this study. Richard Nicholson, Michelle Booth, and Tendai Ngwerume were 594 local principal investigators at the three trial sites. Debra Williams and Hannah Heath from 595 the patient steering group contributed to manual development. Amber Breame, Jade McCune, 
596 and Jan Kelly managed the randomisation. Iskra Potgieter transcribed interview recordings.

597 Anna Frost and Chris Almey contributed to thematic analysis of patient interviews.

598

599 Declaration of conflicts of interest

600 None.

601

602 Funding

603 This paper presents independent research funded by the National Institute for Health

604 Research (NIHR) under its Research for Patient Benefit (RfPB) Programme (Grant Reference

605 Number PB-PG-0613-31106). DAH is an NIHR Senior Investigator. The views expressed are 606 those of the author(s) and not necessarily those of the NHS, the NIHR or the Department of 607 Health and Social Care.

608

609 References

610 - Allen, P. J., Stojadinovic, A., Shriver, C. D., \& Jaques, D. P. (1998). Contributions from

611 surgeons to clinical trials and research on the management of soft tissue sarcoma. Annals

612 of surgical oncology, 5(5), 437-441.

613 - Barkham, M., Mellor-Clark, J., Connell, J., \& Cahill, J. (2006). A core approach to

614 practice-based evidence: A brief history of the origins and applications of the CORE-OM

615 and CORE System. Counselling and psychotherapy research, 6(1), 3-15.

616 - Beecham, J., \& Knapp, M. (2001). Costing psychiatric interventions. Measuring mental

617 health needs, 2, 200-224.

618 - Biggs, J. (2003). Aligning teaching for constructing learning. Higher education academy, $619 \quad 1(4)$ 
620

621 research in psychology, 3(2), 77-101.

622 - Busija, L., Pausenberger, E., Haines, T. P., Haymes, S., Buchbinder, R., \& Osborne, R. H. 623 (2011). Adult measures of general health and health-related quality of life: Medical

624 Outcomes Study Short Form 36-Item (SF-36) and Short Form 12-Item (SF-12) Health

625 Surveys, Nottingham Health Profile (NHP), Sickness Impact Profile (SIP), Medical

626 Outcomes Study Short Form 6D (SF-6D), Health Utilities Index Mark 3 (HUI3), Quality

627 of Well-Being Scale (QWB), and Assessment of Quality of Life (AQOL). Arthritis care

$628 \quad \&$ research, 63(S11), S383-S412.

629

$630 \quad$ theory, research, practice, training, 41(3), 217.

631 - Craig, P., Dieppe, P., Macintyre, S., Michie, S., Nazareth, I., \& Petticrew, M. (2008).

632 Developing and evaluating complex interventions: the new Medical Research Council

633 guidance. British medical journal, 337, a1655.

634 - Davis, A. \& El Refaie A. The epidemiology of tinnitus. In: Tyler R, editor. The

635 Handbook of Tinnitus. Singular; 2000. p. 1-23.

636 - Department of Health. (2009). Provision of Services for Adults with Tinnitus. A Good

637 Practice Guide. London: Central Office of Information.

638 - De Silva, D. (2015). What's getting in the way? Barriers to improvement in the NHS. The 639 Health Foundation.

640 - Evans, C., Connell, J., Barkham, M., Margison, F., McGRATH, G. R. A. E. M. E.,

641 Mellor-Clark, J., \& Audin, K. (2002). Towards a standardised brief outcome measure:

642 Psychometric properties and utility of the CORE-OM. The British journal of psychiatry, $643 \quad 180(1), 51-60$. 
644 - Fackrell, K., Hall, D. A., Barry, J. G., \& Hoare, D. J. (2018). Performance of the Tinnitus

645 Functional Index as a diagnostic instrument in a UK clinical population. Hearing

646 research, $358,74-85$.

647 - Fitzgerald, P., \& Leudar, I. (2010). On active listening in person-centred, solution-

648 focused psychotherapy. Journal of pragmatics, 42(12), 3188-3198.

649 - Fuller, T., Cima, R., Langguth, B., Mazurek, B., Vlaeyen, J. W., \& Hoare, D. J. (2020).

650 Cognitive behavioural therapy for tinnitus. Cochrane database of systematic reviews, (1), 6512020.

652 - Furlong, W. J., Feeny, D. H., Torrance, G. W., \& Barr, R. D. (2001). The Health Utilities 653 Index (HUI®) system for assessing health-related quality of life in clinical studies.

654 Annals of medicine, 33(5), 375-384.

655 - Gander, P. E., Hoare, D.J., Collins, L., Smith, S., \& Hall, D. A. (2011) Tinnitus referral

656 pathways with the National Health Service in England: a survey of their perceived

657 effectiveness among audiology staff. BMC health services research, 11, 162.

658 - Hall, D., Mohamad, N., Firkins, L., Fenton, M., \& Stockdale, D. (2013). Identifying and 659 prioritizing unmet research questions for people with tinnitus: the James Lind Alliance 660 Tinnitus Priority Setting Partnership. Clinical investigation, 3(1), 21-28.

661 - Handscomb, L., Hall, D. A., Hoare, D. J., \& Shorter, G. W. (2016). Confirmatory factor 662 analysis of Clinical Outcomes in Routine Evaluation (CORE-OM) used as a measure of 663 emotional distress in people with tinnitus. Health and quality of life outcomes, 14(1), 124.

664 - Handscomb, L. E., Hall, D. A., Shorter, G. W., \& Hoare, D. J. (2017). Positive and 665 negative thinking in tinnitus: factor structure of the tinnitus cognitions questionnaire. Ear $666 \quad$ and hearing, 38(1), 126. 
667 - Henry, J. A., Stewart, B. J., Abrams, H. B., Newman, C. W., Griest, S., Martin, W. H., et 668 al. (2014). Tinnitus Functional Index (TFI): Development and Clinical Application. 669 Audiology today, 26(Nov/Dec), 40-8.

670 - Hesser, H., Weise, C., Westin, V. Z., \& Andersson, G. (2011). A systematic review and 671 meta-analysis of randomized controlled trials of cognitive-behavioral therapy for tinnitus 672 distress. Clinical psychology review, 31(4), 545-553.

673 - Hoare, D. J., Kowalkowski, V. L., Kang, S., \& Hall, D. A. (2011). Systematic review and 674 meta-analyses of randomized controlled trials examining tinnitus management. 675 Laryngoscope, 121(7), 1555-1564.

676 - Hoare, D. J., Broomhead, E., Stockdale, D., \& Kennedy, V. (2015). Equity and person677 centeredness in provision of tinnitus services in UK National Health Service audiology 678 departments. European journal for person centered healthcare, 3(3), 318-326.

679 - Horvath, A. O., \& Greenberg, L. S. (1989). Development and validation of the Working $680 \quad$ Alliance Inventory. Journal of counseling psychology, 36(2), 223.

681 - McCulloch, A., \& McMurran, M. (2007). The features of a good offender treatment 682 programme manual: A Delphi survey of experts. Psychology, crime \& law, 13(3), 265683274.

684 - Meyer, G., Denham, C. R., \& Battles, J. (2010). Safe practices for better healthcare-2010 685 update: a consensus report. In Washington, DC, National quality forum (p. 58).

686 - Meikle, M. B., Henry, J. A., Griest, S. E., Stewart, B. J., Abrams, H. B., McArdle, R., ... $687 \&$ Folmer, R. L. (2012). The tinnitus functional index: development of a new clinical 688 measure for chronic, intrusive tinnitus. Ear and hearing, 33(2), 153-176.

689 - Miller, T., Deary, V., \& Patterson, J. (2014). Improving access to psychological therapies 690 in voice disorders: a cognitive behavioural therapy model. Current opinion in 691 otolaryngology \& head and neck surgery, 22(3), 201-205. 
692

693

694

695

696

697

698

699

700

701

702

703

704

705

706

707

708

709

710

711

712

713

714

715

716

- Pinto, P. C. L., Marcelos, C. M., Mezzasalma, M. A., Osterne, F. J. V., de Lima, M. D.

M. T., \& Nardi, A. E. (2014). Tinnitus and its association with psychiatric disorders: systematic review. The journal of laryngology \& otology, 128(8), 660-664.

- Race, P. (2014). The lecturer's toolkit: a practical guide to assessment, learning and teaching. Routledge.

- Richmond, H., Hall, A. M., Hansen, Z., Williamson, E., Davies, D., \& Lamb, S. E. (2016). Using mixed methods evaluation to assess the feasibility of online clinical training in evidence based interventions: a case study of cognitive behavioural treatment for low back pain. BMC medical education, 16(1), 163.

- Sanchez, V. A., Hall, D. A., Millar, B., Escabi, C. D., Sharman, A., Watson, J., ... \& Harris, P. (2018). Recruiting ENT and Audiology patients into pharmaceutical trials: Evaluating the multi-centre experience in the UK and USA. International journal of audiology, 57(sup4), S96-S107.

- Stockdale, D., McFerran, D., Brazier, P., Pritchard, C., Kay, T., Dowrick, C., \& Hoare, D. J. (2017). An economic evaluation of the healthcare cost of tinnitus management in the UK. BMC health services research, 17(1), 577.

- Taylor, J. A., Hall, D. A., Walker, D. M., McMurran, M., Casey, A., Stockdale, D., ... \& Hoare, D. J. (2017). A psychologically informed, audiologist-delivered, manualised intervention for tinnitus: protocol for a randomised controlled feasibility trial (Tin Man study). Pilot and feasibility studies, 3(1), 24.

- Thoma, A., Farrokhyar, F., McKnight, L., \& Bhandari, M. (2010). How to optimize patient recruitment. Canadian journal of surgery, 53(3), 205.

- Thompson, D. M., Hall, D. A., Walker, D. M., \& Hoare, D. J. (2017). Psychological therapy for people with tinnitus: a scoping review of treatment components. Ear and hearing, 38(2), 149. 
717 • Thompson, D. M., Taylor, J., Hall, D. A., Walker, D. M., McMurran, M., Casey, A., ... \&

718 Hoare, D. J. (2018). Patients' and clinicians' views of the psychological components of

719 tinnitus treatment that could inform audiologists' usual care: a Delphi survey. Ear and

720 hearing, 39(2), 367.

721 - Tyler, R. S., \& Baker, L. J. (1983). Difficulties experienced by tinnitus sufferers. Journal

722 of speech and hearing disorders, 48(2), 150-154.

723 - Wan, W. S., Mohd, Z. N., Nik, N. A., Azizah, O., Aw, C. L., \& Zuraida, Z. (2015). The

724 effectiveness of psychological interventions among tinnitus sufferers: A review. The

725 medical journal of Malaysia, 70(3), 188-197.

726 - Wilson, P. H., \& Henry J. L. (1998) Tinnitus Cognitions Questionnaire: Development and 727 psychometric properties of a measure of dysfunctional cognitions associated with tinnitus.

728 International tinnitus journal, 4, 23-30.

729

730

731

732

733

734

735

736

737

738

739

740

741 
742 Figure legends.

743 Figure 1. Feasibility trial flow chart

744

745

746

747

748

749

750

751

752

753

754

755

756

757

758

759

760

761

762

763

764

765

766 
767 Table 1. Baseline demographics

\begin{tabular}{llll}
\hline & Psychological & Treatment as usual & Overall (SD) \\
& treatment (SD) & (SD) & \\
\hline Sex & 3 female & 3 female & 6 female \\
& 7 male & 5 male & 12 male \\
Age & $59(10.989)$ & $44(18.330)$ & $53(16.092)$ \\
Baseline TFI & $67.08(23.644)$ & $50.00(23.849)$ & $59.49(23.644)$
\end{tabular}

768

SD = Standard Deviation; TFI = Tinnitus Functional Index. Demographic data for one patient were not provided by trial site
770

771

772

773

774

775

776

777

778

779

780

781

782

783

784

785

786

787

788

789 
790 Table 2. Feasibility outcomes

\begin{tabular}{lcccccc}
\hline & $\begin{array}{c}\text { Site } \\
\mathbf{1}\end{array}$ & $\begin{array}{c}\text { Site } \\
\mathbf{2}\end{array}$ & $\begin{array}{c}\text { Site } \\
\mathbf{3}\end{array}$ & $\begin{array}{c}\text { Psychological } \\
\text { treatment }\end{array}$ & TAU & Total \\
\hline $\begin{array}{l}\text { Recruitment efficiency of those } \\
\text { screened }\end{array}$ & $63 \%$ & $12 \%$ & $*$ & - & - & $*$ \\
\hline Recruitment relative to target & $100 \%$ & $30 \%$ & $60 \%$ & $73 \%$ & $53 \%$ & $63 \%$ \\
\hline Retention of enrolled patients & $30 \%$ & $67 \%$ & $67 \%$ & $64 \%$ & $25 \%$ & $47 \%$ \\
\hline $\begin{array}{l}\text { Primary effectiveness outcome } \\
\text { questionnaire received }\end{array}$ & $100 \%$ & $50 \%$ & $0 \%$ & $43 \%$ & $50 \%$ & $44 \%$ \\
$\begin{array}{l}\text { TAU=Treatment as Usual; * screening data not provided by sites; - = Not applicable. Note the each site had a } \\
\text { recruitment target of 10 patients. }\end{array}$ & & & & &
\end{tabular}

793

793

794

795

796

797

798

799

800

801

802

803

804

805

806

807 
Table 3. Questionnaire data

\begin{tabular}{|c|c|c|c|c|c|c|}
\hline & \multicolumn{2}{|l|}{ Baseline } & \multicolumn{2}{|l|}{ Post-treatment } & \multicolumn{2}{|c|}{ 6-month follow-up } \\
\hline & $\begin{array}{l}\text { Psychological } \\
\text { treatment }\end{array}$ & TAU & $\begin{array}{l}\text { Psychological } \\
\text { treatment }\end{array}$ & TAU & $\begin{array}{l}\text { Psychological } \\
\text { treatment }\end{array}$ & TAU \\
\hline & Mean (SD) & Mean (SD) & Mean (SD) & Mean (SD) & Mean (SD) & Mean (SD) \\
\hline TFI & $67.08(21.669)$ & $50.00(23.849)$ & $26.53(32.027)$ & $8.80(-)$ & $22.48(25.065)$ & $10.40(-)$ \\
\hline TFI-E & $59.00(37.648)$ & $38.75(28.000)$ & $25.56(38.634)$ & $0.00(-)$ & $15.56(21.430)$ & $3.33(-)$ \\
\hline TCQ & $64.67(24.378)$ & $59.00(56.569)$ & 49.67 (12.097) & $56.00(-)$ & $60.00(-)$ & $8.00(-)$ \\
\hline TCQ-N & $46.17(26.164)$ & $48.50(61.518)$ & $32.67(22.811)$ & $36.00(-)$ & $54.00(-)$ & $3.00(-)$ \\
\hline CORE-OM & $8.58(7.425)$ & $6.47(4.575)$ & $2.94(2.080)$ & $1.76(-)$ & $2.94(2.080)$ & $1.47(-)$ \\
\hline WAI-C Task & $74.40(7.162)$ & $80.00(4.243)$ & $79.00(6.928)$ & $83.00(-)$ & & \\
\hline WAI-C Bond & $76.20(8.843)$ & $73.00(15.556)$ & $76.00(6.928)$ & $84.00(-)$ & & \\
\hline WAI-C Goal & 73.40 (7.369) & $80.50(3.536)$ & $78.67(9.238)$ & $75.67(7.234)$ & & \\
\hline WAI-T Task & 38.75 (37.677) & $6.00(-)$ & $75.00(7.071)$ & $50.67(37.846)$ & & \\
\hline WAI-T Bond & 74.50 (9.110) & $84.00(-)$ & $77.50(6.364)$ & $72.00(7.810)$ & & \\
\hline WAI-T Goal & $62.50(11.958)$ & $54.00(-)$ & $73.00(7.071)$ & $54.00(-)$ & & \\
\hline
\end{tabular}

810 Questionnaire; TCQ-N = Tinnitus Cognitions Questionnaire-Negative subscale; CORE-OM = Clinical Outcomes in Routine Evaluation - Outcome Measure; WAI-C = Working 811 Alliance Inventory- 


\section{Background}

2 Tinnitus involves the perception of sound in the absence of any acoustic stimuli (Davis \& El

3 Refaie, 2000), and is often associated with insomnia, hearing problems, depression, and

4 anxiety (Tyler \& Baker, 1983; Pinto et al., 2014). Cognitive behaviour therapy (CBT) has

5 been trialled extensively, but evidence is largely limited to clinical trials where a psychologist

6 delivered it (Fuller et al., 2020; Hesser et al., 2011; Hoare et al., 2011). Some studies have

7 additionally included multiple disciplines such as clinical physicists in audiology, who

8 deliver elements of care that are informed by psychology (e.g. Cima et al., 2012). In the UK

9 most tinnitus care is delivered by audiologists. However, there is no standard protocol for

10 tinnitus management and various approaches to assessment and treatment are used (Hoare et

11 al., 2015). Most audiology departments offer management of hearing loss, use of sound

12 generators, and patient education. Only some departments have access to psychologists

13 (Gander et al., 2011) and there is no evidence for the effectiveness of psychological

14 interventions when delivered by audiologists (Hoare et al., 2011). If audiologists were to use

15 a psychologically informed treatment approach, this could lead to improved outcomes and a 16 more cost-effective service (Stockdale et al., 2017; Wan Suhailah et al., 2015).

18 The Department of Health (now the Department of Health and Social Care) (2009)

19 recommends that audiologists provide psychological therapies where psychologists are not 20 available. Furthermore, patients and clinicians agree that whether CBT delivered by 21 audiologists is an effective treatment for tinnitus is a priority research question (Hall et al., 22 2013). To that end, and in accordance with Medical Research Council (MRC)

23 recommendations for developing and evaluating complex interventions (Craig et al., 2008),

24 we took a phased approach to developing an audiologist delivered intervention that was

25 'psychologically informed'. To begin with, components of psychological therapies for 
26 tinnitus were catalogued in a scoping review (Thompson et al., 2017). The primary aim of 27 that scoping review was to catalogue components of psychological therapies which have been 28 used or tested by psychologists in a format that would inform the development of a 29 standardised audiologist-delivered psychological intervention. Twenty-five 'themes' of 30 components were identified including tinnitus education, psychoeducation, evaluation, 31 treatment rationale, treatment planning, problem solving, behavioural intervention, thought 32 identification, thought challenging, worry time, emotions, social comparison, interpersonal 33 skills, self-concept, lifestyle advice, acceptance and defusion, mindfulness, attention, 34 relaxation, sleep, sound enrichment, comorbidity, treatment reflection, relapse prevention, 35 and common therapeutic skills.

37 In the second development stage, consensus on which therapy components audiologists 38 should deliver as part of their usual care was derived in a three-round Delphi survey of 39 patients who have experienced tinnitus counselling or psychological therapy, and specialist 40 audiologists, hearing therapists, and psychologists, who had significant experience of using 41 counselling or psychotherapeutic techniques for tinnitus (Thompson et al., 2018). The Delphi 42 survey included all those components identified in the scoping review (Thompson et al., 43 2017) and any additional components identified by our experts during the first round of the 44 survey. Components that typically reached consensus for inclusion were common therapeutic 45 skills such as Socratic questioning and active listening, which are common to different 46 therapeutic modalities, rather than specific techniques such as graded exposure therapy or 47 cognitive restructuring, which are unique to a cognitive behavioural orientation. There was 48 clear consensus also to use a psychological model of tinnitus rather than a neurophysiological 49 model in patient education. 
51 Here we report the next stage of this work. The aims were to (1) manualise a low-intensity audiologist-delivered psychologically informed intervention for tinnitus, and (2) evaluate

53

54

55 56

57 feasibility of delivering and evaluating the manualised intervention. The intervention was delivered by NHS audiologists with prior experience in tinnitus management. The study primarily considered feasibility of a clinical trial of the manualised intervention in terms of the likely patient population, willingness of patients to receive/engage with the manualised treatment, willingness of audiologists to recruit and randomise tinnitus patients to a trial, and willingness of patients to be randomised. Secondary objectives considered qualitative judgements on the acceptability of the intervention from audiologist and patient perspectives, and the likely completeness of selected outcome measures in a randomised controlled trial (RCT). It was our aim to embed good practice in patient and public involvement at all stages.

\section{Methods}

The protocol for this work has previously been published (Taylor et al., 2017), and is summarised here.

\section{Development of the manual}

Manual development commenced with a one-day reference group meeting involving steering group and study team members (patients, audiologists, hearing therapists, psychologists, and researchers) to decide on which components to include, and how to incorporate them into a manualised care protocol. It was agreed at this meeting that a working framework for the manual would comprise the following sections: Rationale, Assessment, Education, Treatment Planning/Goal Setting, Management/Self-Management, and Relapse Prevention. All 76 components for which consensus for inclusion was reached in the Delphi survey (Thompson et al., 2018) were first considered for allocation under these headings. This was followed by a 
76 discussion on whether any of the 84 components for which consensus was not reached should

77 be included (no component reached consensus to absolutely exclude) and if so, under which

78 headings they would be written into the manual. Decisions were based on (1) level of

79 agreement from the Delphi survey, (2) whether components had a good evidence-base,

80 theoretical cohesiveness, and, (3) what could realistically be included in a brief low-intensity

81 intervention delivered by audiologists given resource limitations and time-limited training.

82 Individual members of the team drafted specific sections of the manual in accordance with

83 their areas of expertise and according to consensus opinion on what features are typical of a

84 'good' treatment manual (McCulloch \& McMurran, 2007). The manual was then reviewed

85 and amended to reflect a 'whole' in terms of style, coherence, and theory.

87 The final manual included nine sections (see Supplemental Information 1 for the overview

contained in the manual) detailing background and overview of the manual, assessment

through a standardised interview, the rationale for psychologically informed treatment,

90 collaborative goal setting and treatment planning, tinnitus education, management/self-

91 management strategies, relapse prevention, a bibliography containing references related to the

92 intervention and further reading for the audiologist or patient, and appendices containing

93 materials used for training purposes. An accompanying 'toolkit' (listed in Supplemental

94 Information 2) of questionnaires, worksheets, and information leaflets was collated to support

95 use of the manual.

\section{$97 \quad$ Feasibility trial}

98 Ethical approval for the feasibility trial was granted by North West - Preston Research Ethics

99 Committee (reference: 16/NM/0047). The trial involved random allocation of tinnitus

100 patients who received psychologically informed treatment from an audiologist trained in the 
101

102

103

104

105

106

107

108

109

110 Trial sites and training

111 Patients were recruited at three sites, audiology/Ear, Nose and Throat (ENT) services situated

use of the manual, or treatment as usual (TAU) from an audiologist who was not trained in or aware of the content of the treatment manual (Figure 1). Patients were randomised using the randomisation function in Microsoft Excel such that patients allocated to the greatest five random numbers per site were allocated to TAU. A member of the research department not involved in the trial performed randomisation. There was no blinding (to allocation) of the researchers who collected or analysed the qualitative or quantitative data.

\section{***INSERT FIGURE 1 ABOUT HERE***} in (1) Nottingham University Hospitals NHS Trust, (2) Sherwood Forest Hospitals NHS

113 Foundation Trust, and (3) Derby Hospitals NHS Foundation Trust. The audiologists

114 delivering the psychological tinnitus intervention and TAU at each site were senior

115 audiologists with several years of experience of managing tinnitus. A 2-day workshop was 116 developed by AC and DF to train audiologists to deliver the manual. Training was designed

117 to be interactive by using a constructive alignment approach (Biggs, 2003). AC developed

118 lesson plans using the Race lecturer toolkit (Race, 2014) including learning outcomes for 119 each session; resources needed to deliver the session; and the structure, content and method 120 of delivery. One senior audiologist from each site received training in the use of the manual 121 during the workshop. Learning was assessed using the Teach-back technique (Meyer et al., 122 2010).

\section{Participants}


125 The recruitment method targeted people with tinnitus attending each participating site for an 126 audiology assessment appointment as part of the NHS standard care. Following consultation 127 with six NHS audiology sites, it was estimated that on average recruitment of two eligible 128 patients per month per site was readily achievable. We therefore conservatively estimated 129 feasibility of a trial to reflect this and requested each site to recruit and randomise up to ten 130 patients within a 6 month period. For inclusion, patients were required to (1) be adults aged 13118 and over, (2) have intellectual/cognitive capacity to provide informed consent, (3) have 132 sufficient mobility to attend clinics, (4) score $\geq 25$ on the Tinnitus Functional Index (TFI; 133 Meikle et al., 2012), and (5) be willing to complete questionnaires and a semi-structured 134 interview about their experiences of taking part in the trial. Patients were excluded if they (1) 135 had tinnitus with a medically treatable origin, (2) were unable to communicate in English, (3) 136 had comorbid conditions (e.g. dementia) which meant they were unable to give informed 137 consent, or (4) had participated in other tinnitus management research between consenting 138 and the start of the intervention. No exclusions were made because of previous experience 139 with psychological services or medications taken. All patients were enrolled at or before their 140 first visit to audiology with a primary complaint of tinnitus.

142 A member of the research team (JAT) was responsible for recruitment at Site 1. At Site 2 and 143 Site 3, the local PI was delegated responsibility for recruitment, with infrastructure support 144 provided by the National Institute for Health Research (NIHR) Clinical Research Network. 145 JAT regularly monitored recruitment at Sites 2 and 3 and maintained regular email contact 146 with the local PIs to offer support where recruitment rate was lower than required. However, 147 no additional support was requested during the trial. 
150 Psychologically informed treatment was delivered according to the treatment manual. This 151 involved an average of 2.75 sessions (range $=1-3$ ) delivered one-to-one by an audiologist 152 who attended the training workshop.

153

154 Control

155 Patients allocated to the control condition received TAU from an audiologist not trained in 156 psychological therapy and who had not received training in the manualised intervention.

157 TAU typically involved management of hearing loss, education and advice, lasting an 158 average of 1.5 sessions (range $=1-2$ ).

159

160 Outcomes

161 Feasibility of a powered RCT was predefined as (1) a recruitment efficiency of at least 10\%

162 of those screened, (2) recruitment of at least $65 \%$ of the overall target sample size, (3)

163 retention of $80 \%$ of patients who were randomised, (4) completion of $80 \%$ for the primary

164 outcome questionnaires, and (5) patient and clinician compliance to the manualised tinnitus

165 counselling.

166

167 Six questionnaire were completed at each time point. The TFI is a measure of tinnitus

168 symptom severity over the last 2 weeks. It has 25 items (e.g. "Did you feel IN CONTROL in 169 regard to your tinnitus?"), each with a 10-point scale. Scores are summed and divided by 2.5 170 to give a total possible score between 0 and 100. The eight factor TFI has very high internal 171 consistency $(\alpha=0.97)$ (Meikle et al., 2012). It has excellent reliability, stability over time, 172 and sensitivity to individual differences in tinnitus severity, although the auditory subscale 173 (three items) may not contribute to the overall score (Fackrell et al., 2018). 
175 The Tinnitus Cognitions Questionnaire (TCQ; Wilson \& Henry, 1998) consists of a series of 176 statements preceded by the words "I think" or "I tell myself," (e.g., I think "if only the noise 177 would go away"). The first 13 items refer to negative thoughts and the second 13 items refer 178 to positive thoughts. Responses are marked on a five-point Likert scale from "never" = 0, to 179 "very frequently" $=4$. Positive items are reverse scored to give a total score from $0-104$. The 180 TCQ has a reliable two-factor structure and high internal consistency $(\alpha=0.90)$ (Handscomb 181 et al., 2017).

182

183 The Clinical Outcomes in Routine Evaluation - Outcome Measure (CORE-OM; Barkham et 184 al., 2006) is a 34-item (four factor) measure of global distress. Respondents indicate how 185 much each item (e.g. "I have felt optimistic about my future") has applied to them over the 186 past week, using a 5-point Likert scale from "not at all" to "most or all of the time". The CORE-OM includes both positive and negative items (positive items being reverse scored) to 188 give a total score from $0-136$. Initial validation of this four factor questionnaire found it to 189 have high internal consistency ( $\alpha=0.94$ ) (Evans et al., 2002), although validation in a 190 tinnitus population found that a three factor structure provided a better fit to the data 191 (Handscomb et al., 2016).

193 The Health Utilities Index 15Q (HUI; Furlong et al., 2001) is a 15 item measure of health194 related quality of life. Items are multiple choice with 5-6 rank-ordered response options.

195 Decision tables of response combinations are used to determine the health-state level for each 196 domain within the questionnaire, and then using the tables and a scoring algorithm, utility

197 scores for all attributes of health and an overall health-related quality of life score is 198 determined. Overall scores range from $-0.36-1.00$, where $0=$ "death" and $1=$ "perfect 199 health”. Estimates of the reliability of the HUI vary considerably (Busija et al., 2011). 
201 The Working Alliance Inventory (WAI; Horvath \& Greenberg, 1989) provides a measure of 202 the quality of alliance within counselling. It contains 36 items (e.g. "I was worried about the 203 outcome of the sessions") scored on a Likert scale from 1 (Never) to 7 (Always). The WAI 204 contains both positive and negative items, with negative items being reversed scored, giving a 205 possible range of scores from 36 to 252 . Reliability of the three WAI scale scores appear to 206 be high ( $\alpha=0.92,0.92$, and 0.89 respectively) although two subscales ("Tasks" and "Goals") 207 were very highly correlated $(\mathrm{r}=.92)$. 208

209 The Client Service Receipt Inventory (CSRI; Beecham \& Knapp, 2001) is an adaptable form 210 used to collect information on the whole range of services and supports study participants 211 may use. It contains items such as "Please list any use of inpatients hospital services over the 212 last three months".

213

214 After randomisation, and in advance of their first audiology appointment, patients completed 215 the baseline study questionnaires. Questionnaires were completed again immediately at the 216 end of treatment and at follow-up or 6 months after the commencement of treatment 217 (excluding the CSRI which was only completed at follow-up). In practice, some 6-month 218 follow-up questionnaires were completed before the end of treatment. Patients received one 219 reminder (telephone or email) to complete the follow-up questionnaires if they had not been 220 returned within 2 weeks of their due date.

221

222 Treatment fidelity

223 Treatment fidelity required that treatment components were all consistency administered 224 according to the manual. Audiologists recorded administration of treatment components in a 
225 case report form held for each patient, and took part in semi-structured interviews after the 226 last patient had completed their treatment. Interviews were used to determine which 227 components of the manualised care they thought worked well, why, and which components 228 were not useful. Patients involved in the intervention group also took part in semi-structured 229 interviews to discuss their experiences of the intervention. JAT or DMT conducted interviews 230 approximately 4 weeks after the last patient appointment. Clinician interviews were 231 conducted after discharge of their final patient. Interviews were audio-recorded, transcribed 232 verbatim and analysed using a thematic analysis approach (Braun \& Clarke, 2006). DMT, 233 DMW, and two patient representative members of the lead centre's Patient and Public 234 Involvement (PPI) panel conducted analysis of the qualitative data.

235 236

\section{Deviations from protocol}

237 We had originally planned that public research partners trained in interview techniques would 238 conduct patient interviews. However, this ultimately proved unworkable due to the Sponsor's 239 stipulation that they would need formal registration as volunteers with the NHS Trust for this 240 activity. The process for this, involving application and interviews, was deemed too time 241 consuming to be accommodated within the study timeline. We had also originally planned to 242 discuss emerging themes in two focus groups with audiologists and patients, to ensure that 243 the themes and any identified barriers and facilitators to treatment implementation and 244 maintenance of self-management were accurately representative of patient and audiologist 245 experience. However, given the unanticipated length of the treatment process at sites, there 246 was insufficient time for this to be included within the funding period.

\section{Results}

249 Demographic and clinical characteristics 
250 Nineteen patients were recruited to the trial and randomised to manualised care $(n=11)$ or 251 TAU $(n=8)$. Demographic characteristics are reported in Table 1. Mean baseline TFI score 252 was $67.08(\mathrm{SD}=23.644)$. Seven patients scored 25-50 on the TFI, scores interpreted as 253 tinnitus being a significant problem with a possible need for treatment (Henry et al., 2014). 254 Eleven patients scored above 50, interpreted as tinnitus severe enough to qualify for treatment 255 that is more intensive. Demographic data were missing for one patient. 256 257 ***INSERT TABLE 1 ABOUT HERE*** 258 259 Feasibility outcomes

260 Participant flow is presented in Figure 1, and feasibility outcomes are presented in Table 2. 261 Patients were screened from September 2016 to April 2017 (maximum six months from first 262 screen at each site). At Site 1, all 10 participants were recruited within a 3 month period; at 263 Site 2 three patients were recruited within a 3 month period, two of whom were recruited on 264 the same day); at Site 3 six patients were recruited within a 7 week period, five of whom 265 were recruited within the same week). 266

267 Six-month follow-up occurred from April to September 2017. Recruitment efficiency of 268 those screened surpassed the feasibility target at Site 1 where 10 eligible patients were 269 enrolled from just 16 patients screened (63\%), while the target was one enrolment for every 27010 patients screened (10\%). Recruitment efficiency was also met at Site 2, where 24 patients 271 were screened and three patients were recruited (13\%). This difference in recruitment 272 efficiency is in part a consequence of different patient pathways; at Site 1 all patients were 273 referred by their GP to ENT, and from ENT onto audiology. At Site 2 some patients were 274 referred directly to audiology by their GP. Indeed 10 of the 24 patients screened at Site 2 
275 were assessed by the audiologist as requiring referral to ENT or psychiatry and therefore not 276 eligible for the trial. Also at Site 2, one recruited patient failed to attend any further 277 appointments, and three patients screened as eligible declined to participate due to the time 278 commitments involved and geographical distance between home and the audiology service. 279 Site 3 did not return their screening records so we were unable to assess feasibility. 280 281 ***INSERT TABLE 2 ABOUT HERE*** 282 283

Nineteen patients were recruited and consented to take part in the study; representing a total 284 recruitment of $63 \%$ (feasibility target was $65 \%$ ). Site 1 surpassed the target for feasibility 285 with $100 \%$ recruitment. Site 2 recruited three patients $(30 \%)$ and Site 3 recruited six $(60 \%)$. 286

287 288 289 290 291 further $(n=1)$, or commenced other treatment $(n=3)$.

\section{Effectiveness outcomes}

Completeness of questionnaire data is given in Table 2, and descriptive statistics are 296 presented in Table 3. TFI, TCQ, TCQ negative thoughts subscale, and CORE-OM scores 297 decreased between baseline and the end of treatment. TFI scores continued to decrease 298 between the end of treatment and 6-month-follow-up, whereas TCQ and CORE-OM scores were maintained between the end of treatment and 6-month follow-up. TFI emotional distress 300 subscale scores increased between baseline and the end of treatment, but decreased below 301 baseline by 6-month-follow up. Patients and audiologists' scores on the WAI task, bond, and 
302 goal subscales all increased between baseline and end of treatment. No adverse events or 303 adverse reactions were recorded during the trial.

304

305

306

307

308

309

310

311

312

313

314

315

316

317

318

319

320

321

322

323

324

\section{***INSERT TABLE 3 ABOUT HERE***}

\section{Treatment fidelity and experience}

Six themes emerged from thematic analysis of interview transcripts: (1) acceptability of and fidelity to treatment components, (2) factors affecting fidelity, (3) refining treatment components, (4) factors affecting feasibility of wider implementation of treatment, (5) training and supervision, and (6) measurement of tinnitus outcomes.

(1) Acceptability of and fidelity to treatment components.

Patients and audiologists use, acceptance of, and fidelity to the treatment components varied. All audiologists reported that they actively promoted physical activity, and patients reported that they engaged in physical activity during the trial, despite it temporarily increasing awareness of tinnitus. Other components were not used consistently, e.g. one audiologist and one patient (from different sites) stated that they did not formally set any goals for treatment, thereby deviating from the treatment protocol:

“We didn't set any formal goals, it was just a case of, I would go away and see... whether it's got any worse or whether it got any better and in actual fact it's stayed about the same." (Patient 2).

Another audiologist explained that goal setting can be challenging without first engaging in tinnitus education to explain to the patient that they cannot cure their tinnitus: 
325

326

327

328

330

331

332

333

336

337

339

340

341

342

343

344 345

346

347

348

349 “... I find the preliminary bit of goal setting a bit difficult because you've got to start by saying, 'we can't cure your tinnitus, so with that in your mind, what would you like to do? ", (Audiologist 3).

All patients reported being reassured by receiving information about the causes, maintenance, and change in tinnitus over time, and for some patients, education was considered sufficient intervention. For many patients the cognitive behavioural model of tinnitus reflected their personal experiences of tinnitus. Patients and audiologists also commented that the model was understandable:

I think [the cognitive behavioural model is] quite a nice one because it's quite logical, the language that it uses is fairly easy, is nothing too sort of technical or too medical and you can [work] through [the manual] in a logical way." (Audiologist 2)

Relaxation was acceptable to audiologists although it was used sparingly and briefly in and outside of appointments. Patients mostly reported using relaxation to improve sleep. Patients and audiologists also highly valued the inclusion of education about sleep cycles in treatment. Patients in the trial did not use sleep diaries, sleep monitoring devices were favoured, although the principle of monitoring sleep to identify poor sleeping habits was maintained: "No, I've been using [a fitness watch and not the sleep diary] because that records in quite reasonable detail the amount of sleep you've had." (Patient 1)

Cognitive techniques received mixed reviews. Some patients expressed an understanding of avoidance as a mechanism of tinnitus-related distress and recognised when they were engaging in avoidance behaviours:

"I avoided noisy environments. So, like if friends invited me round for a party, I always used to make excuses not to go..." (Patient 3)

Audiologists also demonstrated an understanding of safety behaviours such as avoidance. However, at least one felt unable to recognise avoidance consistently in various contexts: 
350

351

"Safety [avoidance] behaviours...seems very obvious when you read the words.... But then actually when the patient starts talking about the things that they do and their feelings and their attitudes and how they deal with things you don't necessarily think, 'that's a safety behaviour'." (Audiologist 1)

At least one audiologist found thought challenging prompts included in the treatment manual useful, but fidelity to the thought-challenging component varied, often limited to identifying negative thoughts without challenging them:

"I didn't use the thought record sheets. I used the unhelpful thinking styles [material], and patients do find this one quite useful and quite often they'll point out, 'yeah, that's me', and so that helps us to address some of these thoughts." (Audiologist 3)

When thought challenging was used, audiologists either considered the technique ineffective, or believed the benefit would only be short term:

“Most of the time [patients] don't [think of a more helpful realistic thought]. And I don't know whether that's because I'm not enabled enough or whether they just can't do it." (Audiologist 1)

The attention, monitoring and acceptance component of the manual was used in treatment but not consistently or as instructed in the manual. One audiologist introduced the concept of mindfulness, and two used metaphors when they thought it would be acceptable to patients. However, one patient reported their audiologist used metaphors as an imagination exercise rather than as an (intended) defusion technique:
“We didn't go through too many metaphors, obviously the metaphors of aligning the sounds of your tinnitus to natural sounds. We didn't talk about particular river banks." (Patient 1) 
373 Finally, relapse prevention was not addressed according to the manual. Patients remembered

374 being advised to return if their symptoms worsened or if self-management using the

375 techniques learned during the treatment were not effective:

376

"I don't think [the audiologist] did [share the relapse prevention handout] actually, no because I don't think we got on to that point actually." (Patient 1)

378

379 (2) Factors affecting fidelity

380 Audiologists reported a lack of confidence in implementing treatment components including 381 relaxation, acceptance-based components, and goal setting. One suggested that rigid use of 382 the manual would impair the development of the therapeutic relationship, particularly for less 383 experienced audiologists, and in contrast, another felt that the manual would help less experienced audiologists by providing a structure to follow. For one audiologist, confidence reportedly augmented with increasing experience during the trial:

"You're always a little bit nervous to start with. However, I found that after the first few appointments it's just flown. You develop your own pattern in appointments and I guess I developed a pattern very, very quickly and that's because it was very intense training..." (Audiologist 3)

Patients sometimes resisted the use of treatment components such as thought challenging or mindfulness. Audiologists stated their patients would report not having enough time to engage with the treatment outside of appointments, or just believed that the treatment would 393 be unsuccessful. Audiologists also considered some treatment components were within the scope of practice of psychologists or hearing therapists, but not theirs:

"To me [thought challenging] is a logical skill and I don't know that that's my leaning at all. To say to somebody, 'well that thought you are having there is very unhelpful'....if you're a psychologist you could do that. But I can't." (Audiologist 1) 
399 (3) Refining treatment components

400 Patients and audiologists recommended changes to the manual. These included expanding the 401 physical exercise component to include lifestyle advice on diet and alcohol.

"That's something that we do anyway so within the tinnitus therapy process we always talk about lifestyle choices. So, physical exercise comes into that but we also 404 talk about things like diet, we might also talk about you know where patients are 405 maybe drinking excessively." (Audiologist 3) "If they've taken up hobbies that have enabled them to be more distracted, sort of reinforcing those things and said, 'these are the things that you know are going to enable you in the long term to sort of ignore your tinnitus and live with it happily'."

410 One audiologist found that some patients' lack of motivation was a barrier to their 411 engagement in the treatment and that a technique to improve motivation would be useful. “It's like how can we break this down... what happens when you can't motivate a patient... how do you tackle somebody who is clearly giving you excuses...? Cause there's a harsh way which is, 'look, you're just giving lots of excuses.' But that's not the way to do it. That doesn't help anybody. But then is it up to us to go into maybe the deeper reasons why they're doing that, is it a confidence issue [for example], and it's that thing that's stopping that patient from getting better." (Audiologist 3)

418 (4) Factors affecting feasibility of wider implementation of treatment

419 Audiologists unanimously agreed the treatment did not lengthen the amount of time spent 420 with patients throughout their care. However, participation in the intervention arm of the trial 421 affected the usual appointment structure. For example, the audiologists delayed hearing aid 422 fitting to allow time to gather information about underlying mental health problems first. 
423 Audiologists also thought that reducing the time between appointments could increase 424 treatment benefit:

425

“[reducing] follow-up [from] about 12 .. to...6 weeks after the initial appointment... works because patients either take on board everything you say or they struggle with it and if you've kind of let them go for three months you're almost back to square one sometimes." (Audiologist 1)

However, concern was expressed that such changes to appointment structure might not be feasible in audiology services where resources are limited. Continuity of care, with patients seeing the same audiologist throughout their care was also considered beneficial: “.. some patients even though they are hearing aid patients, probably would benefit from being fitted by the tinnitus audiologist rather than an [other] audiologist because some of the reasoning behind why we're fitting the hearing aid has to be related to the tinnitus. And perhaps that was lost ..." (Audiologist 2)

(5) Training and supervision

Audiologists were introduced to the manual across 2 days of training. Although audiologists valued the small group setting, the content of the 2 days was considered overwhelming. Audiologists involved in the trial suggested that it might not be feasible to implement this format outside of the trial for routine clinical practice:

"It was excellent, one-to-one with a group of experts, what else could you ask for? ...Feasibility-wise, would that work in practice? Probably not." (Audiologist 3)

Some audiologists recommended role-play to improve the training to provide practice and feedback on techniques in a range of scenarios in order to help audiologists to understand how components of the manual would work in practice. All audiologists felt that formal 
447

448

449

450

451

452

453

454

455

456

457

458

459

460

461

462

463

464

465

466

467

468

469

470

471

supervision arrangement would be helpful to check adherence to the treatment manual.

However, audiologists also noted the lack of supervision within audiology:

“In adult audiology, we work in a silo... we don't have anyone else except our colleagues to turn to, we don't have any formal supervision like you would if you were a therapist." (Audiologist 3)

The potential for the treatment manual to be used as a training tool for inexperienced audiologists was raised repeatedly during interviews:

"I found [the manual] quite useful in terms of training somebody new to sort of say, 'do you want to delve a bit deeper, is there something in these questions that perhaps is relevant for the patient you've got sat in front of you?" (Audiologist 2)

(6) Measurement of tinnitus outcomes

Patients and audiologists recognised that all the domains measured by the TFI and TCQ could be relevant to different patients, and no additional domains were identified as relevant that were not measured in the trial. The TFI was favoured as an assessment tool by patients and audiologists because of its broad coverage across tinnitus problem domains to enable identification of relevant treatment options. However, one audiologist highlighted that some patients conflate hearing loss and tinnitus when completing the TFI, which may limit its ability to distinguish between tinnitus-related and hearing-related distress. Audiologists were less familiar with the TCQ, although one appreciated it as a means to encourage patients to recognise and talk about their negative thoughts about tinnitus:

"Sometimes patients are quite reluctant to give [negative thoughts], some patients don't like feelings, but to get them to write it down and to score it, you get a bit more of an overview of about where they are and how severe really the tinnitus is and how much it is affecting them psychologically. So that again I've not used that one [TCQ] 
472

473

474

475

476

477

478

479

480

481

482

483

484

485

486

487

488

489

490

491

492

493

494

495

496

routinely but I do think that one is one that I would probably use for those patients where I'm perhaps struggling to see how much is affecting them psychologically." (Audiologist 2)

Patients also welcomed the use of tinnitus questionnaires to monitor their improvement during treatment:

"It makes you start to realise how well you're doing and it gives you a chance to measure that...how bad it is or how you're managing really well." (Patient 2)

Patients and audiologists had different views on the difficulty of completing questionnaires. Whereas audiologists perceived questionnaires to be accessible, patients thought that support should be offered to complete any questionnaires:

"When you give a questionnaire like this and just give it to somebody-and I was put in another room to do it - I think there's an assumption made that everyone can do that task." (Patient 2)

\section{Discussion}

This study was the first to develop and assess feasibility of trialling an audiologist-delivered low intensity psychological intervention for tinnitus. Ultimately, we aimed to provide an evidenced-based, costed, manualised approach to tinnitus care that is attractive to commissioners and represents a flexible and accessible tinnitus treatment. The feasibility trial reported here determined that a manualised psychologically informed treatment was acceptable to patients and audiologists, but that it is not yet feasible to test in a multi-site effectiveness trial. Recruitment reached only 19 patients (63\%) after 6 months, just short of the $65 \%$ target for feasibility, while average retention for the duration of the trial reached only nine patients $(47 \%)$, very short of the $80 \%$ target for feasibility. Patient recruitment is acknowledged to be one of the most difficult and least predictable elements of a clinical trial. 
497 (Allen et al., 1998; Thoma et al., 2010; Sanchez et al., 2018). Here, performance varied 498 across the three sites. The best in terms of recruitment efficiency and recruitment rate was

499 Site 1, an audiology service based in a large teaching hospital where staff are supported to be 500 research active. Having a large pool of potentially eligible patients to draw on, as well as 501 engaging with audiologists who have previous experience in participating in RCTs, are 502 known factors that can help to achieve study targets for hearing trials (Sanchez et al., 2018).

503 In addition, a member of the research team was responsible for recruitment at Site 1, and did 504 not have to contend with the same clinic demands as PIs at the other sites. That said, the 505 pattern of recruitment would suggest all could have reached feasibility. Site 3 in particular 506 recruited five patients (50\% of target) within a single week, suggesting competing demands 507 and/or a lack of engagement with the research, rather than low patient numbers, were barriers 508 to recruitment at that site. In contrast, Site 1 performed poorest in terms of patient retention 509 indicating that even experienced participating sites need a risk mitigation plan and regular 510 trial monitoring. We would concur with the recommendations suggested by Sanchez et al. 511 (2018) that a future RCT needs to ensure that it has sufficient resource to support a carefully

512 specified plan to promote recruitment and retention of tinnitus patients. Furthermore, based

513 on current findings, we would suggest that treatment fidelity could be improved and more

514 accurately monitored by (1) including an audiologist familiarisation phase of treatment

515 implementation before including patients in analyses, and (2) including direct in-session

516 observation of a random selection of treatment sessions using standardised fidelity evaluation

517 forms, based on the treatment manual.

518

519 From the Delphi survey (Thompson et al., 2018) we know that psychoeducation and common

520 therapeutic skills are considered more important than specific CBT techniques in

521 psychological tinnitus treatment. Patients and audiologists considered it essential to include 
522 psychoeducation and common therapeutic skills (aimed at informing the patient and 523 conveying empathy) in psychological tinnitus treatment. Giving patients information about 524 common cognitive distortions was considered essential, but cognitive restructuring was not.

525 This pattern suggests that patients and audiologists understood the contribution of

526 maladaptive behaviours and negative thoughts to bothersome tinnitus, but did not think that 527 the audiologist's role should or could extend to managing patients' use of specific cognitive 528 behavioural techniques. This issue was further highlighted in the current trial. Interviews 529 revealed audiologists chose not to use certain psychological treatment components from the 530 manual because (1) they lacked confidence in using them as training was brief and 531 insufficient for them to learn how to implement them safely and effectively, (2) they thought 532 patients do not want to record, monitor, and challenge specific negative thoughts and 533 behaviours, and (3) they believed certain components would be ineffective. More work is 534 therefore required to consider what audiologists can and want to deliver either within their 535 current or a revised clinic and appointment structure, and what training and supervision 536 arrangements need to be in place for these skills to be developed, used, and maintained 537 throughout a clinical trial. Beyond feasibility and evaluation of effectiveness, it will also be 538 essential to explore how the intervention can be implemented into routine clinical practice, 539 which will involve further challenges such as organisational development, workplace cultures 540 and individual barriers (De Silva, 2015).

541

542 Similar issues are being faced in other areas. For example, there is emerging evidence that 543 CBT-enhanced voice therapy for functional dysphonia leads to greater improvement in 544 general well-being and distress (Miller, Deary, \& Patterson, 2014), but as with the current 545 study there are open questions about how clinicians should be trained and supervised and 546 how cost-effective this approach may be. There are examples in other fields of how training 
547 might be achieved on a large scale. Richmond et al. (2016) evaluated feasibility of an online

548 programme to train physiotherapists in the use of cognitive behavioural approaches to the

549 management of non-specific low back pain. They found an internet-based approach to

550 training to be equivalent to face-to-face group training in terms of retention of theoretical and

551 procedural knowledge. However, clinicians who received the face-to-face training showed

552 greater self-efficacy in delivering the assessment component of the therapy, were more

553 satisfied with their training, and were more 'psychosocially' rather than 'biomedically'

554 orientated after training, and by implication were more aligned with a cognitive behavioural

555 approach. Regardless, uptake of the treatment approach after training was similar across both

556 groups (about one third). Their conclusion, that there is a need to develop strategies for

557 training and support to deliver treatments that use cognitive behavioural approaches, read

558 relevant to all allied health professionals at this time. In the context of the current study,

559 issues such as the acceptability and preference for different training formats will need to be

560 explored.

561

562 Certain revisions to the TinMan study manual and training require consideration before it can

563 be subjected to further feasibility testing. These include additional information about tinnitus

564 and psychoeducation, including information about negative thought patterns and avoidant

565 behaviours. An appreciation of patient preference and need with respect to psychological

566 treatment components was also apparent in the current study. Specific cognitive behavioral

567 techniques tend to purposefully engage patients in initially unwanted, though temporary,

568 thoughts or emotional states. It is of importance to train audiologists in how to deal with these

569 challenges and ultimately make use of the patient-states resulting from these approaches. The

570 natural resistance and transferal of distress in both patients and audiologists when using these 
571 techniques needs to be adequately covered in training that balances informed patient choice 572 and need.

573

\section{Conclusion}

575 What emerged most strongly from this study is that an audiologist-delivered low-intensity 576 psychological intervention is an acceptable approach to tinnitus management, but even very 577 experienced audiologists require more extensive training to 'upskill' in the use of 578 psychological treatment components. Audiologists involved in delivering the psychological 579 treatment reported that the amount of training was insufficient and lacked role-play and 580 feedback from a supervisor to increase understanding of how to deliver the treatment 581 effectively. This needs to be addressed before further testing. One potentially cost-effective 582 means of training audiologists may be to develop reusable learning objects, e.g. including 583 video recordings of role-play scenarios where an audiologist is using a set of psychological 584 techniques from the manual. Once training is addressed, the treatment may progress to further 585 feasibility testing. There has been recent discussion in the UK audiology community about 586 some form of compulsory continual professional development or qualification in tinnitus care 587 for practicing audiologists. The manual developed here, and the training resources that will 588 support its use, could potentially meet this perceived need. 589

\section{Acknowledgements}

591 We acknowledge the support of the National Institute for Health Research Clinical Research 592 network (CRN) in participant recruitment. Nottingham University Hospitals NHS Trust was 593 the Sponsor for this study. Richard Nicholson, Michelle Booth, and Tendai Ngwerume were 594 local principal investigators at the three trial sites. Debra Williams and Hannah Heath from 595 the patient steering group contributed to manual development. Amber Breame, Jade McCune, 
596 and Jan Kelly managed the randomisation. Iskra Potgieter transcribed interview recordings.

597 Anna Frost and Chris Almey contributed to thematic analysis of patient interviews.

598

599 Declaration of conflicts of interest

600 None.

601

$602 \quad$ Funding

603 This paper presents independent research funded by the National Institute for Health

604 Research (NIHR) under its Research for Patient Benefit (RfPB) Programme (Grant Reference

605 Number PB-PG-0613-31106). DAH is an NIHR Senior Investigator. The views expressed are

606 those of the author(s) and not necessarily those of the NHS, the NIHR or the Department of

607 Health and Social Care.

608

609 References

610 - Allen, P. J., Stojadinovic, A., Shriver, C. D., \& Jaques, D. P. (1998). Contributions from

611 surgeons to clinical trials and research on the management of soft tissue sarcoma. Annals

612 of surgical oncology, 5(5), 437-441.

613 - Barkham, M., Mellor-Clark, J., Connell, J., \& Cahill, J. (2006). A core approach to

614 practice-based evidence: A brief history of the origins and applications of the CORE-OM

615 and CORE System. Counselling and psychotherapy research, 6(1), 3-15.

616 - Beecham, J., \& Knapp, M. (2001). Costing psychiatric interventions. Measuring mental

617 health needs, 2, 200-224.

618 - Biggs, J. (2003). Aligning teaching for constructing learning. Higher education academy, $619 \quad 1(4)$ 
620

621 research in psychology, 3(2), 77-101.

622 - Busija, L., Pausenberger, E., Haines, T. P., Haymes, S., Buchbinder, R., \& Osborne, R. H. 623 (2011). Adult measures of general health and health-related quality of life: Medical

624 Outcomes Study Short Form 36-Item (SF-36) and Short Form 12-Item (SF-12) Health

625 Surveys, Nottingham Health Profile (NHP), Sickness Impact Profile (SIP), Medical

626 Outcomes Study Short Form 6D (SF-6D), Health Utilities Index Mark 3 (HUI3), Quality

627 of Well-Being Scale (QWB), and Assessment of Quality of Life (AQOL). Arthritis care

$628 \quad \&$ research, 63(S11), S383-S412.

629

$630 \quad$ theory, research, practice, training, 41(3), 217.

631 - Craig, P., Dieppe, P., Macintyre, S., Michie, S., Nazareth, I., \& Petticrew, M. (2008).

632 Developing and evaluating complex interventions: the new Medical Research Council

633 guidance. British medical journal, 337, a1655.

634 - Davis, A. \& El Refaie A. The epidemiology of tinnitus. In: Tyler R, editor. The

635 Handbook of Tinnitus. Singular; 2000. p. 1-23.

636 - Department of Health. (2009). Provision of Services for Adults with Tinnitus. A Good

637 Practice Guide. London: Central Office of Information.

638 - De Silva, D. (2015). What's getting in the way? Barriers to improvement in the NHS. The 639 Health Foundation.

640 - Evans, C., Connell, J., Barkham, M., Margison, F., McGRATH, G. R. A. E. M. E.,

641 Mellor-Clark, J., \& Audin, K. (2002). Towards a standardised brief outcome measure:

642 Psychometric properties and utility of the CORE-OM. The British journal of psychiatry, $643 \quad 180(1), 51-60$. 
644 - Fackrell, K., Hall, D. A., Barry, J. G., \& Hoare, D. J. (2018). Performance of the Tinnitus

645 Functional Index as a diagnostic instrument in a UK clinical population. Hearing

646 research, $358,74-85$.

647 - Fitzgerald, P., \& Leudar, I. (2010). On active listening in person-centred, solution-

648 focused psychotherapy. Journal of pragmatics, 42(12), 3188-3198.

649 - Fuller, T., Cima, R., Langguth, B., Mazurek, B., Vlaeyen, J. W., \& Hoare, D. J. (2020).

650 Cognitive behavioural therapy for tinnitus. Cochrane database of systematic reviews, (1),

6512020.

652 - Furlong, W. J., Feeny, D. H., Torrance, G. W., \& Barr, R. D. (2001). The Health Utilities

653 Index (HUI®) system for assessing health-related quality of life in clinical studies.

$654 \quad$ Annals of medicine, 33(5), 375-384.

655 - Gander, P. E., Hoare, D.J., Collins, L., Smith, S., \& Hall, D. A. (2011) Tinnitus referral

656 pathways with the National Health Service in England: a survey of their perceived

657 effectiveness among audiology staff. BMC health services research, 11, 162.

658 - Hall, D., Mohamad, N., Firkins, L., Fenton, M., \& Stockdale, D. (2013). Identifying and 659 prioritizing unmet research questions for people with tinnitus: the James Lind Alliance

660 Tinnitus Priority Setting Partnership. Clinical investigation, 3(1), 21-28.

661 - Handscomb, L., Hall, D. A., Hoare, D. J., \& Shorter, G. W. (2016). Confirmatory factor

662 analysis of Clinical Outcomes in Routine Evaluation (CORE-OM) used as a measure of 663 emotional distress in people with tinnitus. Health and quality of life outcomes, 14(1), 124.

664 - Handscomb, L. E., Hall, D. A., Shorter, G. W., \& Hoare, D. J. (2017). Positive and 665 negative thinking in tinnitus: factor structure of the tinnitus cognitions questionnaire. Ear $666 \quad$ and hearing, 38(1), 126. 
667 - Henry, J. A., Stewart, B. J., Abrams, H. B., Newman, C. W., Griest, S., Martin, W. H., et 668 al. (2014). Tinnitus Functional Index (TFI): Development and Clinical Application. 669 Audiology today, 26(Nov/Dec), 40-8.

670 - Hesser, H., Weise, C., Westin, V. Z., \& Andersson, G. (2011). A systematic review and 671 meta-analysis of randomized controlled trials of cognitive-behavioral therapy for tinnitus 672 distress. Clinical psychology review, 31(4), 545-553.

673 - Hoare, D. J., Kowalkowski, V. L., Kang, S., \& Hall, D. A. (2011). Systematic review and 674 meta-analyses of randomized controlled trials examining tinnitus management. 675 Laryngoscope, 121(7), 1555-1564.

676 - Hoare, D. J., Broomhead, E., Stockdale, D., \& Kennedy, V. (2015). Equity and person677 centeredness in provision of tinnitus services in UK National Health Service audiology 678 departments. European journal for person centered healthcare, 3(3), 318-326.

679 - Horvath, A. O., \& Greenberg, L. S. (1989). Development and validation of the Working 680 Alliance Inventory. Journal of counseling psychology, 36(2), 223.

681 - McCulloch, A., \& McMurran, M. (2007). The features of a good offender treatment 682 programme manual: A Delphi survey of experts. Psychology, crime \& law, 13(3), 265683274.

684

- Meyer, G., Denham, C. R., \& Battles, J. (2010). Safe practices for better healthcare-2010 685 update: a consensus report. In Washington, DC, National quality forum (p. 58).

686 - Meikle, M. B., Henry, J. A., Griest, S. E., Stewart, B. J., Abrams, H. B., McArdle, R., ... 687 \& Folmer, R. L. (2012). The tinnitus functional index: development of a new clinical 688 measure for chronic, intrusive tinnitus. Ear and hearing, 33(2), 153-176.

689 - Miller, T., Deary, V., \& Patterson, J. (2014). Improving access to psychological therapies 690 in voice disorders: a cognitive behavioural therapy model. Current opinion in 691 otolaryngology \& head and neck surgery, 22(3), 201-205. 
692

693

694

695

696

697

698

699

700

701

702

703

704

705

706

707

708

709

710

711

712

713

714

715

716

- Pinto, P. C. L., Marcelos, C. M., Mezzasalma, M. A., Osterne, F. J. V., de Lima, M. D.

M. T., \& Nardi, A. E. (2014). Tinnitus and its association with psychiatric disorders: systematic review. The journal of laryngology \& otology, 128(8), 660-664.

- Race, P. (2014). The lecturer's toolkit: a practical guide to assessment, learning and teaching. Routledge.

- $\quad$ Richmond, H., Hall, A. M., Hansen, Z., Williamson, E., Davies, D., \& Lamb, S. E. (2016). Using mixed methods evaluation to assess the feasibility of online clinical training in evidence based interventions: a case study of cognitive behavioural treatment for low back pain. BMC medical education, 16(1), 163.

- Sanchez, V. A., Hall, D. A., Millar, B., Escabi, C. D., Sharman, A., Watson, J., ... \& Harris, P. (2018). Recruiting ENT and Audiology patients into pharmaceutical trials: Evaluating the multi-centre experience in the UK and USA. International journal of audiology, 57(sup4), S96-S107.

- Stockdale, D., McFerran, D., Brazier, P., Pritchard, C., Kay, T., Dowrick, C., \& Hoare, D. J. (2017). An economic evaluation of the healthcare cost of tinnitus management in the UK. BMC health services research, 17(1), 577.

- Taylor, J. A., Hall, D. A., Walker, D. M., McMurran, M., Casey, A., Stockdale, D., ... \& Hoare, D. J. (2017). A psychologically informed, audiologist-delivered, manualised intervention for tinnitus: protocol for a randomised controlled feasibility trial (Tin Man study). Pilot and feasibility studies, 3(1), 24.

- Thoma, A., Farrokhyar, F., McKnight, L., \& Bhandari, M. (2010). How to optimize patient recruitment. Canadian journal of surgery, 53(3), 205.

- Thompson, D. M., Hall, D. A., Walker, D. M., \& Hoare, D. J. (2017). Psychological therapy for people with tinnitus: a scoping review of treatment components. Ear and hearing, 38(2), 149. 
717 • Thompson, D. M., Taylor, J., Hall, D. A., Walker, D. M., McMurran, M., Casey, A., ... \& 718 Hoare, D. J. (2018). Patients' and clinicians' views of the psychological components of 719 tinnitus treatment that could inform audiologists' usual care: a Delphi survey. Ear and 720 hearing, 39(2), 367.

721

- Tyler, R. S., \& Baker, L. J. (1983). Difficulties experienced by tinnitus sufferers. Journal 722 of speech and hearing disorders, 48(2), 150-154.

723 - Wan, W. S., Mohd, Z. N., Nik, N. A., Azizah, O., Aw, C. L., \& Zuraida, Z. (2015). The 724 effectiveness of psychological interventions among tinnitus sufferers: A review. The 725 medical journal of Malaysia, 70(3), 188-197.

726

- Wilson, P. H., \& Henry J. L. (1998) Tinnitus Cognitions Questionnaire: Development and 727 psychometric properties of a measure of dysfunctional cognitions associated with tinnitus. 728 International tinnitus journal, 4, 23-30.

729

730

731

732

733

734

735

736

737

738

739

740

741 
742 Figure legends.

743 Figure 1. Feasibility trial flow chart

744

745

746

747

748

749

750

751

752

753

754

755

756

757

758

759

760

761

762

763

764

765

766 
767 Table 1. Baseline demographics

\begin{tabular}{llll}
\hline & Psychological & Treatment as usual & Overall (SD) \\
& treatment (SD) & (SD) & \\
\hline Sex & 3 female & 3 female & 6 female \\
& 7 male & 5 male & 12 male \\
Age & $59(10.989)$ & $44(18.330)$ & $53(16.092)$ \\
Baseline TFI & $67.08(23.644)$ & $50.00(23.849)$ & $59.49(23.644)$
\end{tabular}

768

SD = Standard Deviation; TFI = Tinnitus Functional Index. Demographic data for one patient were not provided by trial site

770

771

772

773

774

775

776

777

778

779

780

781

782

783

784

785

786

787

788

789 
790 Table 2. Feasibility outcomes

\begin{tabular}{|c|c|c|c|c|c|c|}
\hline & $\begin{array}{c}\text { Site } \\
1\end{array}$ & $\begin{array}{c}\text { Site } \\
2\end{array}$ & $\begin{array}{c}\text { Site } \\
3\end{array}$ & $\begin{array}{l}\text { Psychological } \\
\text { treatment }\end{array}$ & TAU & Total \\
\hline $\begin{array}{l}\text { Recruitment efficiency of those } \\
\text { screened }\end{array}$ & $63 \%$ & $12 \%$ & $*$ & - & - & $*$ \\
\hline Recruitment relative to target & $100 \%$ & $30 \%$ & $60 \%$ & $73 \%$ & $53 \%$ & $63 \%$ \\
\hline Retention of enrolled patients & $30 \%$ & $67 \%$ & $67 \%$ & $64 \%$ & $25 \%$ & $47 \%$ \\
\hline $\begin{array}{l}\text { Primary effectiveness outcome } \\
\text { questionnaire received }\end{array}$ & $100 \%$ & $50 \%$ & $0 \%$ & $43 \%$ & $50 \%$ & $44 \%$ \\
\hline
\end{tabular}

793

793

794

795

796

797

798

799

800

801

802

803

804

805

806

807 
Table 3. Questionnaire data

\begin{tabular}{|c|c|c|c|c|c|c|}
\hline & \multicolumn{2}{|l|}{ Baseline } & \multicolumn{2}{|l|}{ Post-treatment } & \multicolumn{2}{|c|}{ 6-month follow-up } \\
\hline & $\begin{array}{l}\text { Psychological } \\
\text { treatment }\end{array}$ & TAU & $\begin{array}{l}\text { Psychological } \\
\text { treatment }\end{array}$ & TAU & $\begin{array}{l}\text { Psychological } \\
\text { treatment }\end{array}$ & TAU \\
\hline & Mean (SD) & Mean (SD) & Mean (SD) & Mean (SD) & Mean (SD) & Mean (SD) \\
\hline TFI & $67.08(21.669)$ & $50.00(23.849)$ & $26.53(32.027)$ & $8.80(-)$ & $22.48(25.065)$ & $10.40(-)$ \\
\hline TFI-E & $59.00(37.648)$ & $38.75(28.000)$ & $25.56(38.634)$ & $0.00(-)$ & $15.56(21.430)$ & $3.33(-)$ \\
\hline TCQ & $64.67(24.378)$ & $59.00(56.569)$ & 49.67 (12.097) & $56.00(-)$ & $60.00(-)$ & $8.00(-)$ \\
\hline TCQ-N & $46.17(26.164)$ & $48.50(61.518)$ & $32.67(22.811)$ & $36.00(-)$ & $54.00(-)$ & $3.00(-)$ \\
\hline CORE-OM & $8.58(7.425)$ & $6.47(4.575)$ & $2.94(2.080)$ & $1.76(-)$ & $2.94(2.080)$ & $1.47(-)$ \\
\hline WAI-C Task & $74.40(7.162)$ & $80.00(4.243)$ & $79.00(6.928)$ & $83.00(-)$ & & \\
\hline WAI-C Bond & $76.20(8.843)$ & $73.00(15.556)$ & $76.00(6.928)$ & $84.00(-)$ & & \\
\hline WAI-C Goal & 73.40 (7.369) & $80.50(3.536)$ & $78.67(9.238)$ & $75.67(7.234)$ & & \\
\hline WAI-T Task & 38.75 (37.677) & $6.00(-)$ & $75.00(7.071)$ & $50.67(37.846)$ & & \\
\hline WAI-T Bond & 74.50 (9.110) & $84.00(-)$ & $77.50(6.364)$ & $72.00(7.810)$ & & \\
\hline WAI-T Goal & $62.50(11.958)$ & $54.00(-)$ & $73.00(7.071)$ & $54.00(-)$ & & \\
\hline
\end{tabular}

810 Questionnaire; TCQ-N = Tinnitus Cognitions Questionnaire-Negative subscale; CORE-OM = Clinical Outcomes in Routine Evaluation - Outcome Measure; WAI-C = Working 811 Alliance Inventory- 


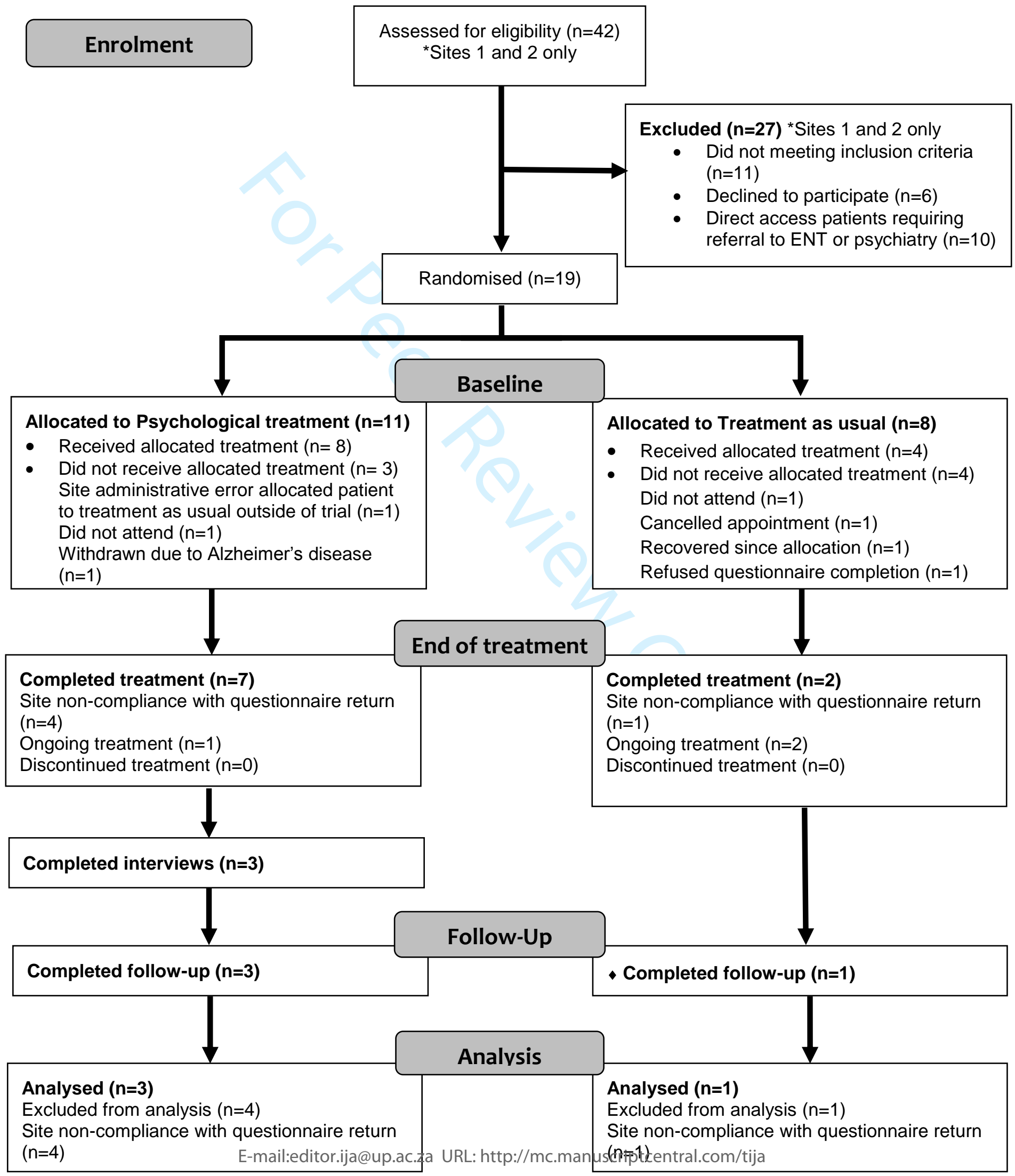




\section{Supplemental Information 1. OVERVIEW OF MANUAL}

The materials in this manual are for delivery to NHS patients with intrusive tinnitus by an audiologist. It requires use of core counselling skills within the context of relationship building between audiologist and patient working in partnership with one another in a therapeutic alliance. Beginning with assessment, using the Relational Skills Model (more detail in Appendices, Section 9), the audiologist works in partnership with the patient through the following process:

\begin{tabular}{|c|c|c|}
\hline $\begin{array}{l}\text { PHASES OF RELATIONSHIP } \\
\text { DEVELOPMENT }\end{array}$ & PROCESS & SKILLS \\
\hline Setting up the relationship & $\begin{array}{l}\text { Contacting/meeting the patient } \\
\text { Getting to know the patient } \\
\text { Communicating with \& } \\
\text { contracting with the patient }\end{array}$ & $\begin{array}{l}\text { Initial core skills: } \\
\text { Attending skills } \\
\text { Active listening skills \& } \\
\text { contracting skills }\end{array}$ \\
\hline Developing the relationship & $\begin{array}{l}\text { Developing the relationship } \\
\text { Problem identification \& } \\
\text { assessment }\end{array}$ & $\begin{array}{l}\text { Additional core skills: } \\
\text { Presence \& communication } \\
\text { of the core conditions } \\
\text { Paraphrasing } \\
\text { Summarising } \\
\text { Identifying \& reflecting } \\
\text { feelings, content \& meaning } \\
\text { Asking questions }\end{array}$ \\
\hline Working with the relationship & $\begin{array}{l}\text { Challenging \& creating new } \\
\text { meaning, different possibilities } \\
\text { \& perspectives }\end{array}$ & $\begin{array}{l}\text { Enhanced skills: } \\
\text { The skill of challenging - } \\
\text { Confronting } \\
\text { Use of self-disclosure, } \\
\text { immediacy \& silence } \\
\text { Clarifying } \\
\text { Reassessing } \\
\text { Probing } \\
\text { Giving feedback \& sharing } \\
\text { information }\end{array}$ \\
\hline The established relationship & $\begin{array}{l}\text { Clarifying \& focusing on likely } \\
\text { changes } \\
\text { Working collaboratively to } \\
\text { make plans, set goals, \& } \\
\text { consider \& evaluate possible } \\
\text { strategies \& directions }\end{array}$ & $\begin{array}{l}\text { Intuitive \& learned skills: } \\
\text { Deeper empathy } \\
\text { Focusing } \\
\text { Use of metaphor \& hunches } \\
\text { Drawing together themes } \\
\text { Clarifying \& identifying goals } \\
\text { Action planning }\end{array}$ \\
\hline $\begin{array}{l}\text { Maintaining and ending the } \\
\text { relationship }\end{array}$ & $\begin{array}{l}\text { Implementing \& maintaining } \\
\text { change } \\
\text { Supporting self-management } \\
\text { strategies }\end{array}$ & $\begin{array}{l}\text { Embedding skills: } \\
\text { Encouragement, support \& } \\
\text { affirmations } \\
\text { Review, monitor, evaluate \& } \\
\text { facilitate ending } \\
\text { Signposting/referring on }\end{array}$ \\
\hline
\end{tabular}

Midwinter R, Dixon J (2015) Embedding Counselling and Communication Skills. A Relational Skills Model. Routledge, Hove: p. 3 
The process and skills used (more detail below) allow for the British Society of Audiology (BSA) Practice Guidance: Common Principles of Rehabilitation for Adults in Audiology Services to be fulfilled. These are:

- Identifying individual needs

- Setting joint goals

- Making shared informed decisions

- Supporting self-management

\section{CORE COUNSELLING SKILLS}

\section{Attending and listening}

- Active listening

- Listening with purpose and responding in such a way that patients are aware they have been heard and understood

\section{Reflective skills}

- Restating what you believe to be a significant word or phrase the patient has used

- Paraphrasing involves conveying the patient's core messages (facts and feelings) in your own words

- Summarising is offering the patient a précis of the information they have given you not as a list of details and facts but as an organised overview of important themes or cluster of concerns

Reflective skills:

- Capture what a patient is telling you and repeat the message in your own words

- Are valuable for 'tracking' patients, since they impose minimal direction from the practitioner

- Enable the practitioner to communicate the core values, to clarify and to acknowledge the patient's experiences

- Build relationships that are supportive and challenging

- Are ideal information-gathering skills

\section{Probing skills}

- Questioning to facilitate exploration

- Making statements is a gentle form of probing. For example, instead of asking a patient, "What did she do to upset you?", you might say, "I'm not sure what she did to upset you". Statements tend to be less intrusive and controlling than questions

Probing skills:

- Tend to focus on what the practitioner wants to know and not necessarily on what the patient wants to tell

- Lead or direct patients

- Help to obtain information from the patient

- Influence direction of the exploration

- Increase practitioner control over process and content 
- Should be used with care because of the above, especially in the early stages

\section{STRUCTURE OF MANUAL}

As shown in the Table of Contents (page 2), the process of treatment comprises six broad sections:

- Patient assessment

- Rationale for psychologically informed treatment

- Goal setting and treatment planning

- Patient education

- Patient management/self-management (with eight sub sections)

- Relapse prevention

At the end of Patient Assessment (Section 2) there are recommendations about which elements of Patient Education (Section 5) and Patient Management/Self-Management (Section 6) to focus on dependent on individual needs. However, it is recommended that Rapid Relaxation (Section 6.2) should be a core (non-optional) element of Patient Management/Self-Management.

If it is agreed that the patient would benefit from psychologically informed treatment, it is important to discuss the Rationale for Psychologically informed Treatment (Section 3) with them prior to Goal Setting and Treatment Planning (Section 4). Having worked through relevant elements of Sections 5 and 6, conclude the treatment with Relapse Prevention (Section 7).

The Bibliography (Section 8) contains references related to the intervention, training resources and further reading. The Appendices (Section 9) contain materials used to train audiologists in the use of the manual.

\section{TOOLKIT OF RESOURCES}

The manual is supported by a separate toolkit booklet (and electronic files) comprising resources to aid the delivery of tinnitus management and enable learning by the patient as s/he works through the process in partnership with the audiologist. Guidance for what resources to use and when to use them is highlighted in red throughout the manual. You are expected to print/photocopy specific resources from the toolkit as required for each patient. 


\section{Supplemental Information 2. TOOLKIT OF RESOURCES}

T1 TINNITUS FUNCTIONAL INDEX ( \& SCORING RUBRIC)

T2 TINNITUS CASE HISTORY QUESTIONNAIRE

T3 FORMULATION WORKSHEET (for Sections 3 and 6.3)

T4 BTA LEAFLET ALL ABOUT TINNITUS VER.1.4

T5 COGNITIVE MODEL OF TINNITUS PSYCHOLOGY TOOL

T6 STAGES OF CHANGE MODEL

T7 GOAL SETTING SHEET

T8 ACTION PLANNING SHEET

T9 GOAL SETTING \& ACTION PLANNING PRACTICE FRAMEWORK

T10 WHAT KEEPS TINNITUS GOING PSYCHOLOGY TOOL

T11 WHAT CAUSES TINNITUS PSYCHOLOGY TOOL

T12 MEANING IN TINNITUS PSYCHOLOGY TOOL

T13 BTA LEAFLET TINNITUS AND STRESS VER.1.4

T14 RELAXATION TRAINING DIARY

T15 FEAR \& AVOIDANCE EXAMPLE FORMULATION

T16 EXPOSURE WORKSHEET

T17 NEGATIVE THINKING EXAMPLE FORMULATION

T18 UNHELPFUL THINKING STYLES SHEET

T19 THOUGHT RECORD - BLANK COPY

T20 THOUGHT RECORD - EXAMPLE COPY

T21 PHYSICAL EXERCISE EXAMPLE FORMULATION

T22 PHYSICAL EXERCISE DIARY

T23 SLEEP CYCLES SHEET

T24 SLEEP DIARY

T25 BTA LEAFLET TAMING TINNITUS VER.1.3

T26 ACCEPTANCE IN METAPHORS SHEET

T27 MINDFULNESS: THREE SIMPLE WAYS TO GET PRESENT SHEET

T28 MY 'MANAGING TINNITUS' BLUEPRINT: MAKING A PLAN FOR WELLBEING 